\title{
Appendices
}

\section{APPENDIX 1.1}

Based on data from the OECD, we created a (admittedly crude) measurement of needs-adjusted social spending figures in the policy domains of unemployment benefits and old-age pensions. In a first step, unemployment spending and pension spending were divided by the unemployment rate and the proportion of people above 65 years of age, respectively. In a second step, the needs-adjusted spending figures were standardized (as in, divided by their standard deviations), so that they can be compared on a similar scale. During this process, we followed the definitions provided by the OECD:

Public unemployment spending is defined as expenditure on cash benefits for people to compensate for unemployment. This includes redundancy payments from public funds, as well as the payment of pensions to beneficiaries before they reach the standard pensionable age, if these payments are made because the beneficiaries are out of work or for other labour market policy reasons. This indicator is measured in percentage of GDP.

Unemployment rate is the number of unemployed people as a percentage of the labour force, where the latter consists of the unemployed plus those in paid or self-employment. Unemployed people are those who report that they are without work, that they are available for work and that they have taken active steps to find work in the previous four weeks.

Public pension spending is defined as all cash expenditures (including lumpsum payments) on old-age and survivors' pensions. Old-age cash benefits provide an income for persons retired from the labour market or guarantee incomes when a person has reached a 'standard' pensionable age or fulfilled the necessary contributory requirements. This category also includes early retirement pensions: pensions paid before the beneficiary has reached the 'standard' pensionable age relevant to the programme. It excludes programmes concerning early retirement for labour market reasons. Old age benefits also include social expenditure on services for elderly people, 
such as day care and rehabilitation services, home-help services and other benefits in kind. It also includes expenditure on the provision of residential care in an institution. This indicator is measured in percentage of GDP.

The elderly population is defined as people aged 65 and over. This indicator is measured as a percentage of the population.

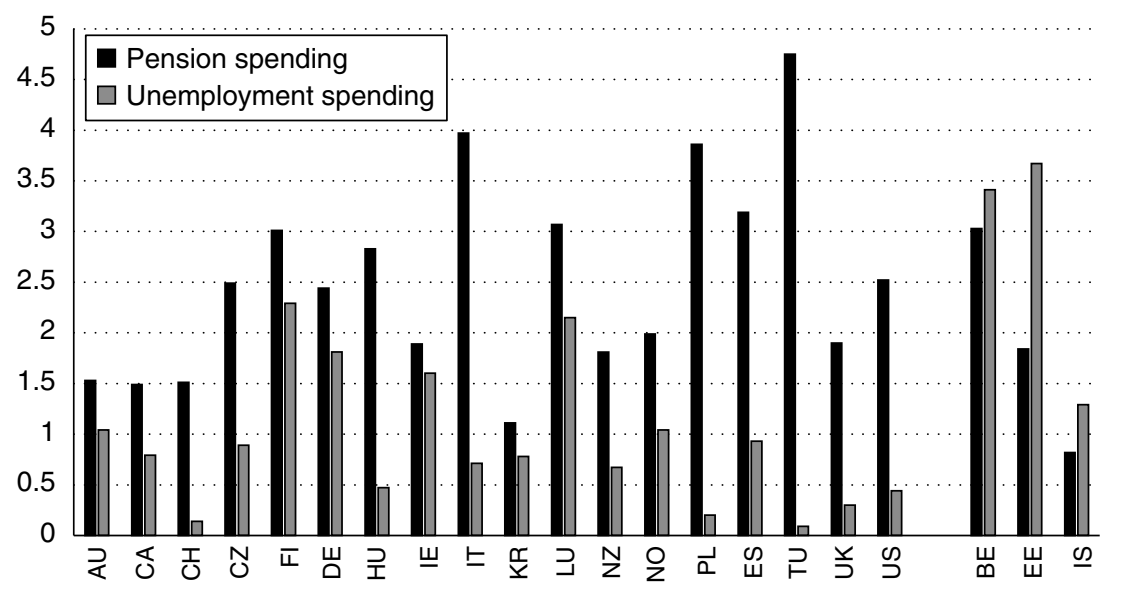

Note: We included only those OECD countries for which data were available for the four different indicators: public unemployment spending, public pension spending, unemployment rate and elderly population. We chose 2014 because it was the most recent year for which data were available for the four indicators.

Countries are: AU Australia; CA Canada; CH Switzerland; CZ Czech Republic; FI Finland; DE Germany; HU Hungary; IE Ireland; IT Italy; KR Korea; LU Luxembourg; NZ New Zealand; NO Norway; PL Poland; ES Spain; TU Turkey; UK United Kingdom; US United States; BE Belgium; EE Estonia and IS Iceland.

Figure A1.1.1 Needs-adjusted pensions and unemployment spending, 2014 


\section{APPENDIX 2.1}

Table A2.1.1 The relative importance of deservingness dimensions in vignette-based experiments embedded in public opinion surveys

\begin{tabular}{|c|c|c|c|c|}
\hline & Country & $\begin{array}{l}\text { Social welfare } \\
\text { domain }\end{array}$ & $\begin{array}{l}\text { Dependent } \\
\text { variable }\end{array}$ & Deservingness dimensions \\
\hline $\begin{array}{l}\text { Cook } \\
(1979)\end{array}$ & $\begin{array}{l}\text { United } \\
\text { States } \\
\text { (Chicago) }\end{array}$ & $\begin{array}{l}\text { General social } \\
\text { services }\end{array}$ & $\begin{array}{l}\text { Support index } \\
\text { (ranging from } \\
\text { sympathy for } \\
\text { the vignette } \\
\text { to willingness } \\
\text { to pay higher } \\
\text { taxes for social } \\
\text { programmes) }\end{array}$ & $\begin{array}{l}\text { 1. severity of disability* } \\
\text { 2. reason for condition* } \\
\text { 3. severity of poverty* } \\
\text { 4. age* }\end{array}$ \\
\hline $\begin{array}{l}\text { Groskind } \\
(1991) \\
\text { Iyengar } \\
(1990) \\
\text { Will (1993) }\end{array}$ & $\begin{array}{l}\text { United } \\
\text { States }\end{array}$ & $\begin{array}{l}\text { Public } \\
\text { assistance }\end{array}$ & $\begin{array}{l}\text { Net benefit } \\
\text { amount for: } \\
\text { mother-only } \\
\text { and two-parent } \\
\text { families } \\
\text { Mother-only } \\
\text { families }\end{array}$ & $\begin{array}{l}\text { 1. number of children* } \\
\text { 2. father's work status* } \\
\text { 3. family's weekly income* } \\
\text { 4. mother's work status* } \\
\text { mother's education } \\
\text { 1. number of children* } \\
\text { 2. family's weekly income* } \\
\text { 3. father's work status* } \\
\text { mother's work status } \\
\text { mother's education } \\
\text { 1. father's work status* } \\
\text { 2. family's weekly income* } \\
\text { 3. number of children* } \\
\text { 4. mother's work status* }\end{array}$ \\
\hline $\begin{array}{l}\text { Appelbaum } \\
(2001)\end{array}$ & $\begin{array}{l}\text { United } \\
\text { States }\end{array}$ & $\begin{array}{l}\text { General } \\
\text { welfare } \\
\text { policies }\end{array}$ & $\begin{array}{l}\text { Likelihood of } \\
\text { recommending } \\
\text { liberal policies/ } \\
\text { no benefits }\end{array}$ & $\begin{array}{l}\text { 1. deservingness* } \\
\text { 2. attribution of } \\
\text { responsibility* } \\
\text { adherence to } \\
\text { mainstream norms } \\
\text { race }\end{array}$ \\
\hline $\begin{array}{l}\text { DeSante } \\
\text { (2013) }\end{array}$ & $\begin{array}{l}\text { United } \\
\text { States }\end{array}$ & $\begin{array}{l}\text { Welfare } \\
\text { assistance }\end{array}$ & $\begin{array}{l}\text { The desired } \\
\text { amount of } \\
\text { welfare } \\
\text { assistance }\end{array}$ & $\begin{array}{l}\text { 1. work history* } \\
\text { 2. race* }\end{array}$ \\
\hline $\begin{array}{l}\text { Hjorth } \\
(2016)\end{array}$ & Sweden & Child benefits & $\begin{array}{l}\text { Approval } \\
\text { of the right } \\
\text { of intra-EU } \\
\text { migrants }\end{array}$ & $\begin{array}{l}\text { 1. children's country of } \\
\text { residence* } \\
\text { 2. nationality* } \\
\text { 3. number of children* }\end{array}$ \\
\hline
\end{tabular}


Table A2.1.1 (continued)

\begin{tabular}{|c|c|c|c|c|}
\hline & Country & $\begin{array}{l}\text { Social welfare } \\
\text { domain }\end{array}$ & $\begin{array}{l}\text { Dependent } \\
\text { variable }\end{array}$ & Deservingness dimensions \\
\hline & & & $\begin{array}{l}\text { to receive } \\
\text { Swedish child } \\
\text { benefit }\end{array}$ & \\
\hline \multirow[t]{2}{*}{$\begin{array}{l}\text { Kootstra } \\
\text { (2016) }\end{array}$} & $\begin{array}{l}\text { United } \\
\text { Kingdom } \\
\text { and the } \\
\text { Netherlands }\end{array}$ & $\begin{array}{l}\text { General } \\
\text { welfare } \\
\text { policies }\end{array}$ & $\begin{array}{l}\text { Deservingness } \\
\text { of financial } \\
\text { support from } \\
\text { government }\end{array}$ & \\
\hline & & & $\begin{array}{l}\text { United } \\
\text { Kingdom }\end{array}$ & $\begin{array}{l}\text { 1. job-seeking efforts* } \\
\text { 2. ethnicity* } \\
\text { 3. family status* } \\
\text { 4. work history* } \\
\text { 5. migration status* } \\
\text { financial support from } \\
\text { relatives* } \\
\text { 6. type of job* } \\
\text { 7. gender* } \\
\text { 1. job-seeking efforts* } \\
\text { 2. work history } \\
\text { 3. migration status* } \\
\text { 4. family status* } \\
\text { 5. financial support from } \\
\text { relatives* } \\
\text { 6. type of job* } \\
\text { gender }\end{array}$ \\
\hline $\begin{array}{l}\text { van Der Aa } \\
\text { et al. } \\
(2017 \text {, } \\
2018)\end{array}$ & $\begin{array}{l}\text { The } \\
\text { Netherlands }\end{array}$ & Healthcare & $\begin{array}{l}\text { Forced choice } \\
\text { between two } \\
\text { care-seeking } \\
\text { individuals in a } \\
\text { given scenario: } \\
\text { who deserves } \\
\text { the allocation } \\
\text { of collectively } \\
\text { financed } \\
\text { healthcare } \\
\text { resources most? }\end{array}$ & $\begin{array}{l}\text { 1. severity of illness* } \\
\text { 2. financial resources* } \\
\text { 3. lifestyle prior to onset of } \\
\text { illness* } \\
\text { behaviour during } \\
\text { treatment* } \\
\text { 4. health insurance } \\
\text { premium* }\end{array}$ \\
\hline $\begin{array}{l}\text { Reeskens } \\
\text { and van der } \\
\text { Meer } \\
(2017 \text {, } \\
\text { 2019) }\end{array}$ & $\begin{array}{l}\text { The } \\
\text { Netherlands }\end{array}$ & $\begin{array}{l}\text { Unemploy- } \\
\text { ment } \\
\text { benefits }\end{array}$ & $\begin{array}{l}\text { Desired level } \\
\text { of replacement } \\
\text { rate }\end{array}$ & $\begin{array}{l}\text { 1. job-seeking behaviour* } \\
\text { 2. reason for being } \\
\text { unemployed* } \\
\text { 3. country of origin* } \\
\text { 4. length of residence* } \\
\text { 5. labour market } \\
\text { consistency* }\end{array}$ \\
\hline
\end{tabular}


Table A2.1.1 (continued)

\begin{tabular}{|c|c|c|c|c|}
\hline & Country & $\begin{array}{l}\text { Social welfare } \\
\text { domain }\end{array}$ & $\begin{array}{l}\text { Dependent } \\
\text { variable }\end{array}$ & Deservingness dimensions \\
\hline & & & & $\begin{array}{l}\text { salary* } \\
\text { number of children* } \\
\text { age* } \\
\text { 6. } \begin{array}{l}\text { motivation for } \\
\text { migration* }\end{array}\end{array}$ \\
\hline Buss (2020) & Germany & $\begin{array}{l}\text { Unemploy- } \\
\text { ment } \\
\text { benefits }\end{array}$ & $\begin{array}{l}\text { Benefit } \\
\text { conditions }\end{array}$ & $\begin{array}{l}\text { 1. child-caring } \\
\text { responsibilities* } \\
\text { 2. job-seeking efforts* } \\
\text { 3. reason for } \\
\text { unemployment* } \\
\text { 4. age* } \\
\text { 5. ethnic background* } \\
\text { 1. age* } \\
\text { 2. child-caring } \\
\text { responsibilities* } \\
\text { 3. ethnic background* } \\
\text { reason for } \\
\text { unemployment } \\
\text { job-seeking efforts } \\
\text { 1. job-seeking efforts* } \\
\text { 2. reason for } \\
\text { unemployment* } \\
\text { 3. age* } \\
\text { ethnic background } \\
\text { child-caring } \\
\text { responsibilities }\end{array}$ \\
\hline $\begin{array}{l}\text { Castillo et } \\
\text { al. (2019) }\end{array}$ & Chile & $\begin{array}{l}\text { Old-age } \\
\text { pensions }\end{array}$ & $\begin{array}{l}\text { Just pension } \\
\text { amount }\end{array}$ & $\begin{array}{l}\text { 1. number of years in } \\
\text { workforce* } \\
\text { last salary* } \\
\text { 2. current pension income } \\
\text { 3. gender* } \\
\text { number of dependants* } \\
\text { number of children } \\
\text { raised } \\
\text { ethnic/social background } \\
\text { educational level }\end{array}$ \\
\hline
\end{tabular}

Note: The numbers indicate the relative importance of the deservingness dimensions in explaining support for social welfare in terms of the (standardized) effect sizes on the outcome variables. If no asterisks $(*)$ are shown, the deservingness dimension had no significant effect on the outcome $(\mathrm{p}>0.05)$. 


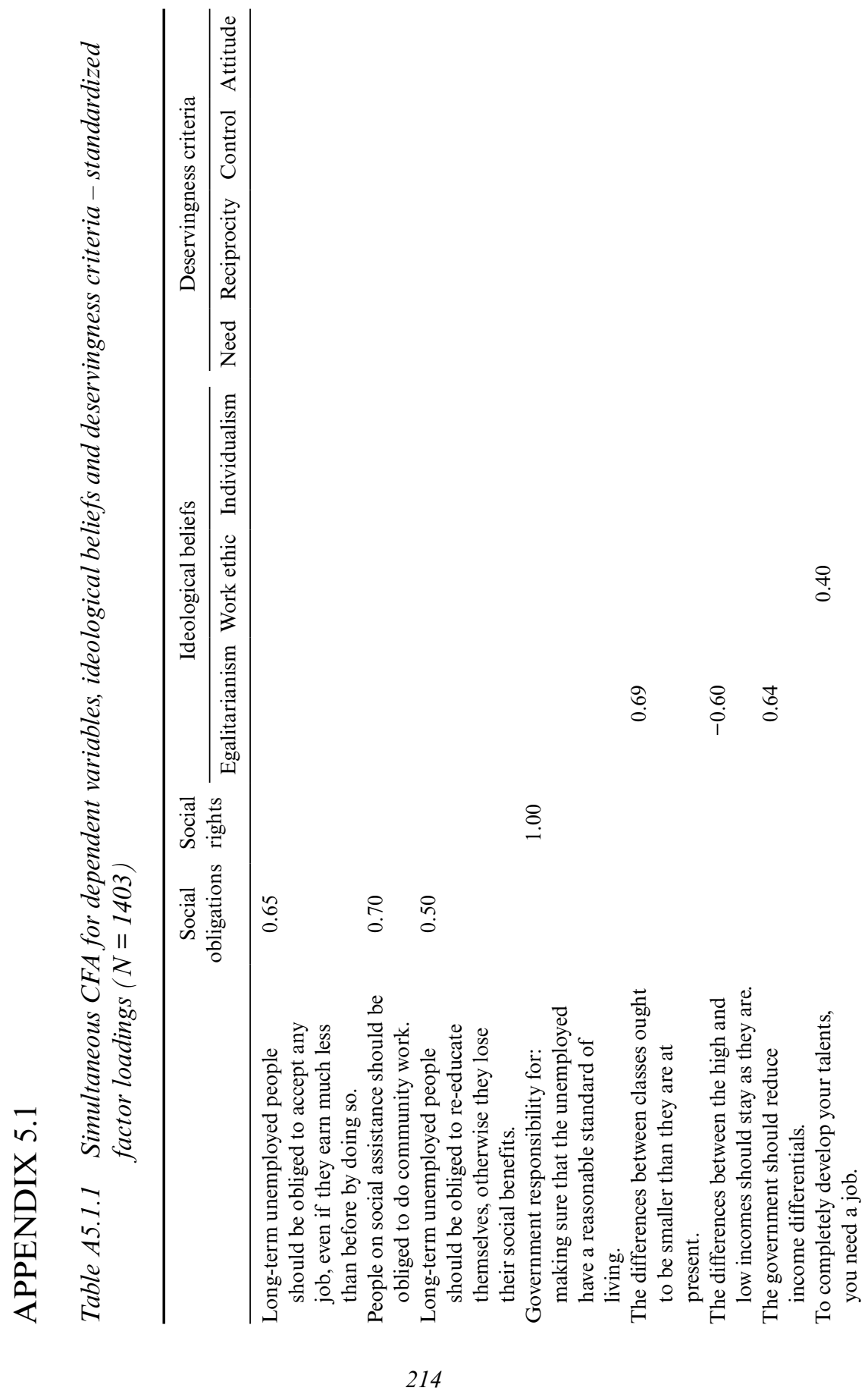




$$
\begin{aligned}
& \text { in } \stackrel{0}{0} \stackrel{a}{0} \\
& \text { ڤ. }
\end{aligned}
$$$$
\stackrel{0}{\stackrel{0}{1}}
$$$$
\text { 寺路: }
$$

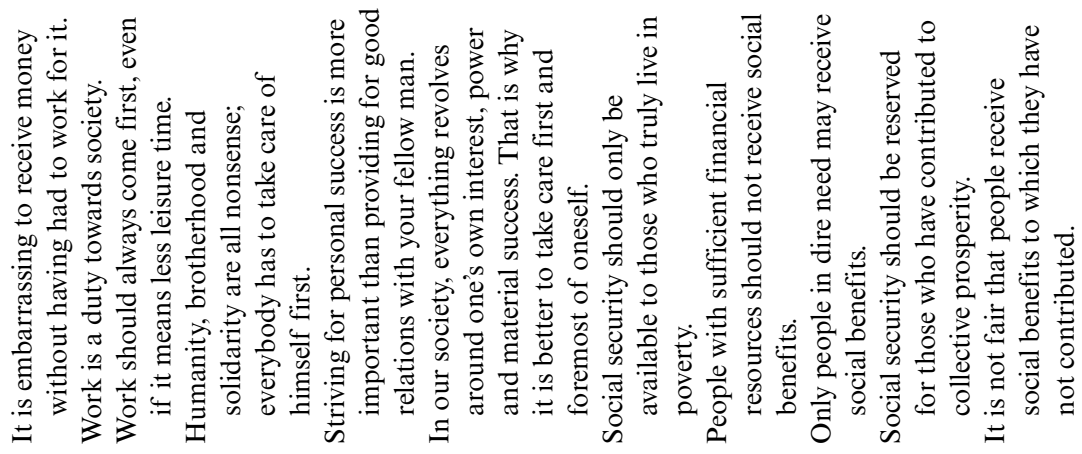




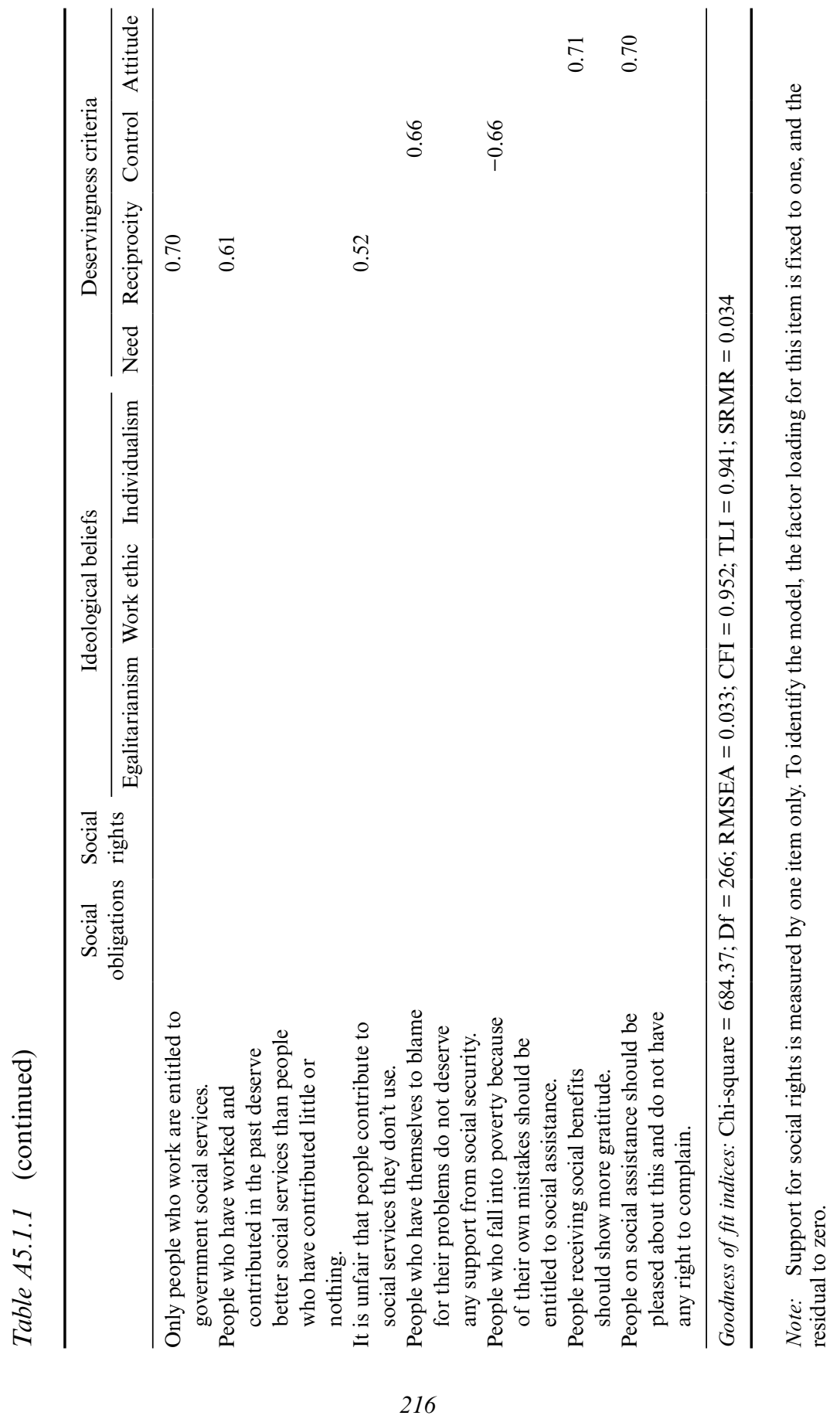




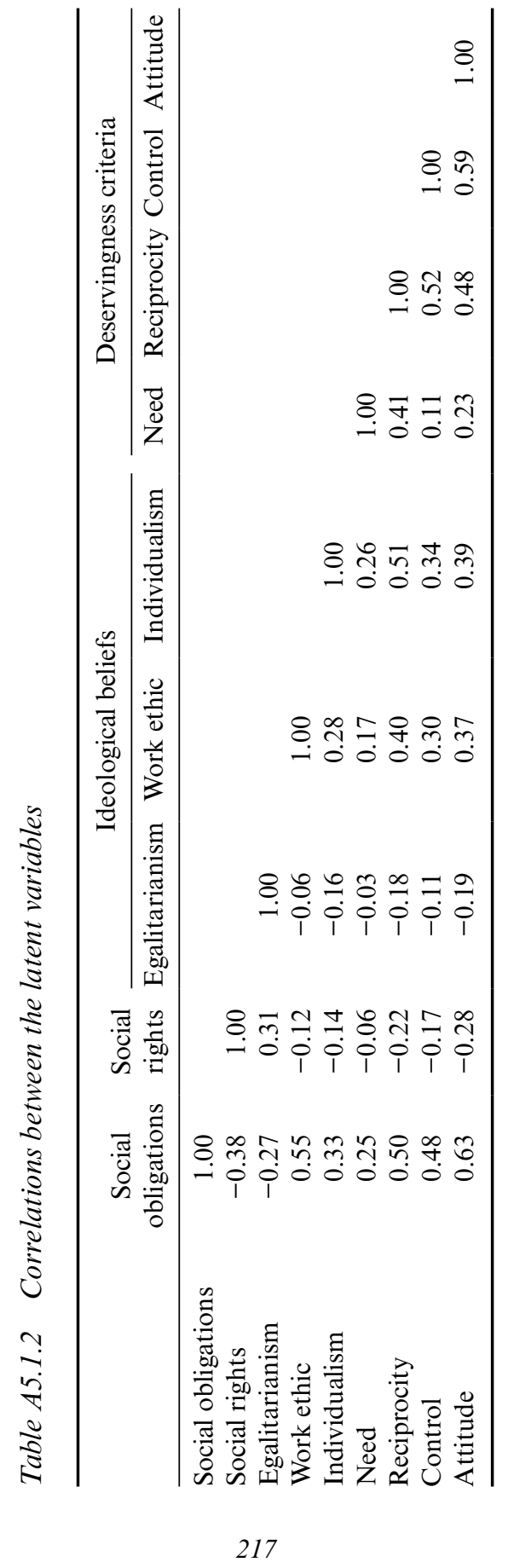




\section{APPENDIX 6.1}

In each country, four relatively homogeneous focus groups consisting of about five to ten participants were formed using the following recruitment guidelines:

Middle class: completed higher education (i.e., three years or more), an income level equivalent to 150 per cent of national median wage for a fulltime worker over 25 , homeownership for the majority of the participants, maximum two students or unemployed, variation in their marital status (e.g., married/partner, single parent, parent with primary school children, parent with teenage children, empty nest parents and persons without children).

Working class: less than three years higher education, the presence of at least four persons in the group without higher education, household income level below the third decile (e.g., under $£ 348$ weekly for the United Kingdom), maximum two students or unemployed and variation in marital status.

Old-age pensioners: age 62 to 75 years (with majority age 67+), variation in pension benefit level (with at least two persons on lowest pension level/ minimum pension), sufficient hearing as a condition for participation, some participants from single-person households (e.g., widowed, divorced) and some childless participants or with children who live far away.

Young adults: age 18 to 35 (with the ideal aim to achieve as even an age distribution as possible), three students, at least three parents and with some variation in family status.

Furthermore, some common criteria for all groups were agreed upon: a gender balance, exclusion of politicians and persons working as market analysts; a balance between left-wing and right-wing participants; and inclusion of members of ethnic minorities. Table A6.1.1 reports for each country separately the list of participants from all focus groups with their main sociodemographic characteristics. Unfortunately, there was an unexpectedly large number of cancellations and non-show ups in Denmark, which is why most of the Danish focus groups only had five participants. 


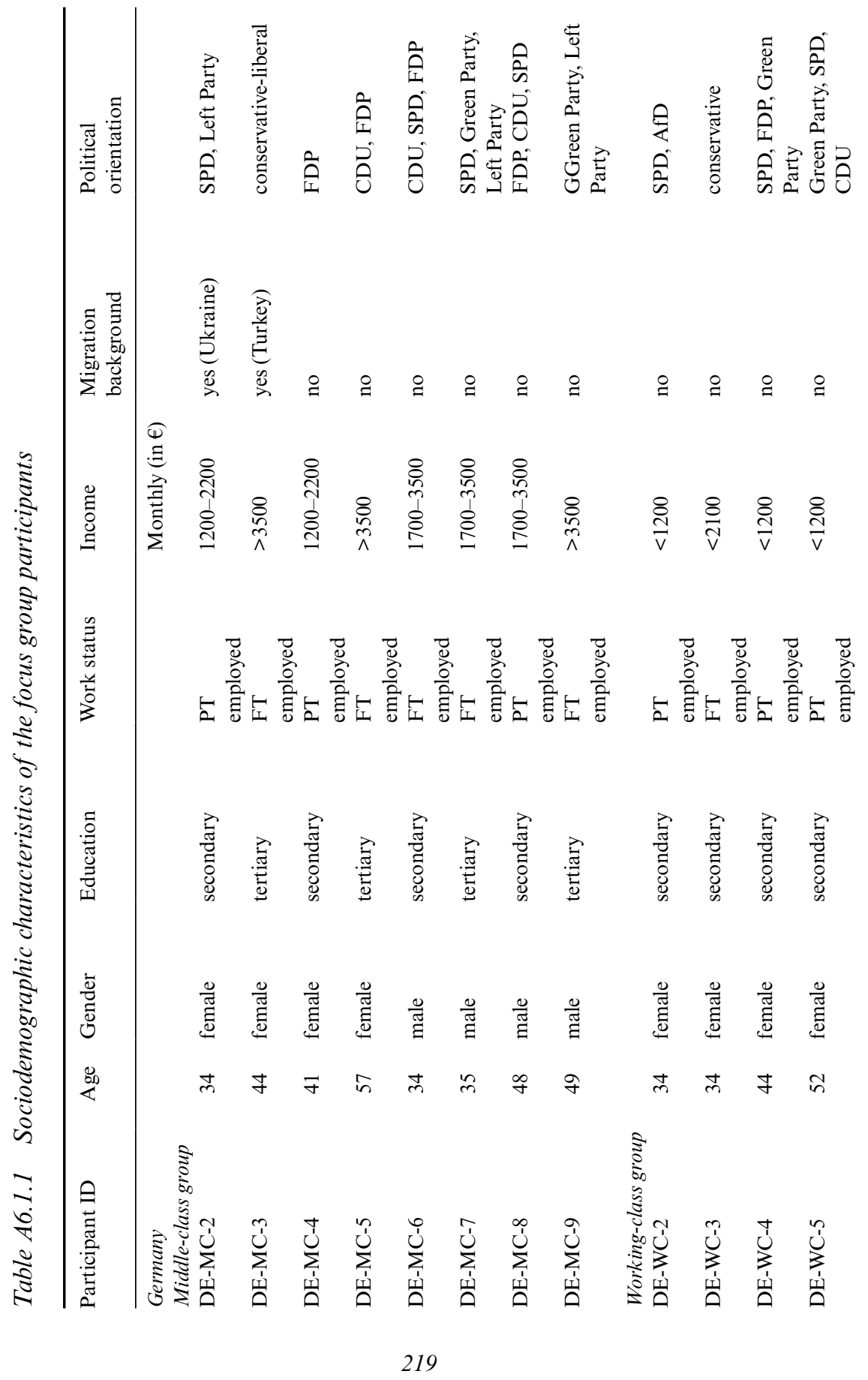

Tijs Laenen - 9781839101892 


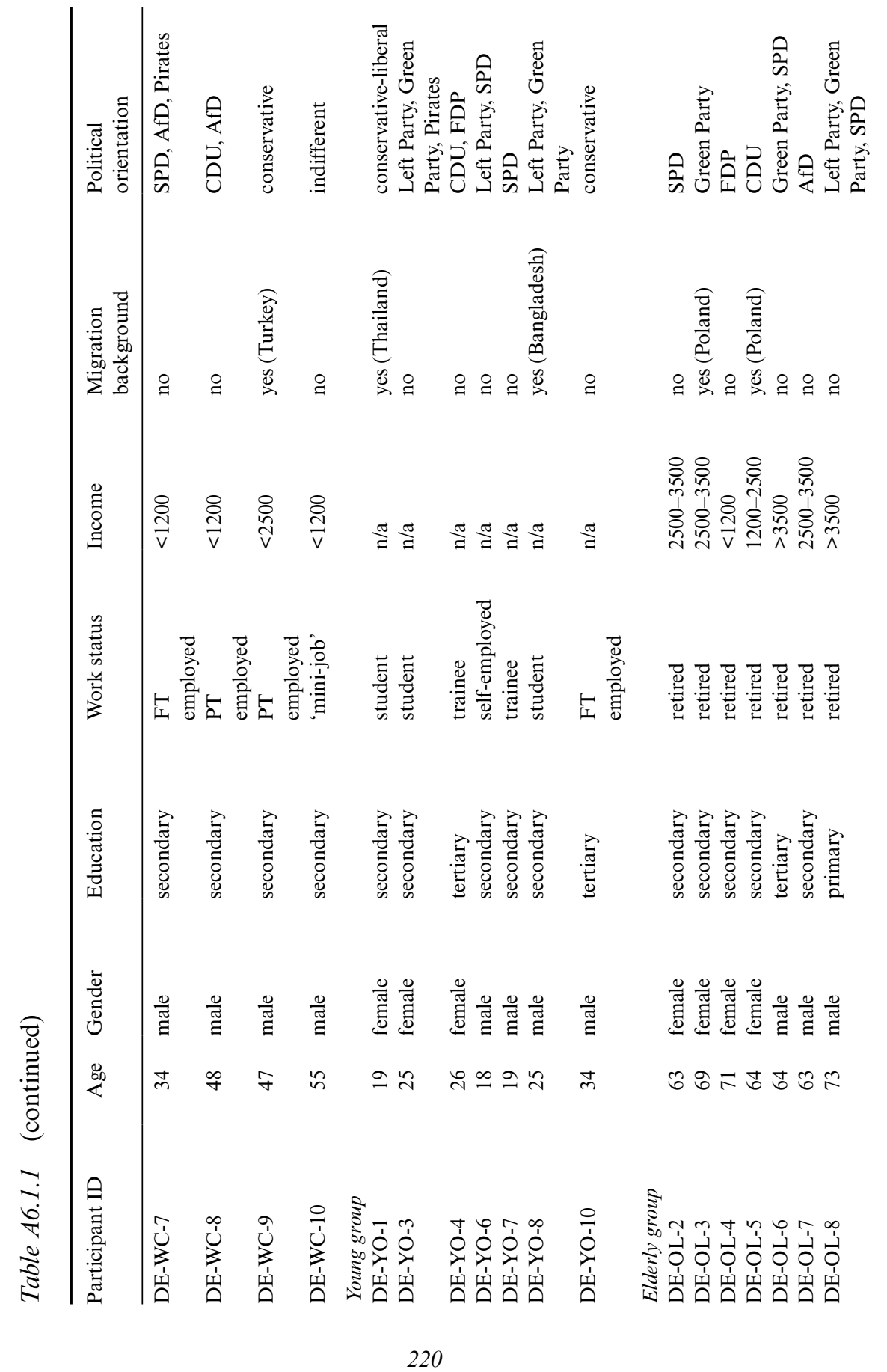




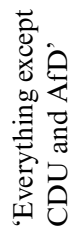

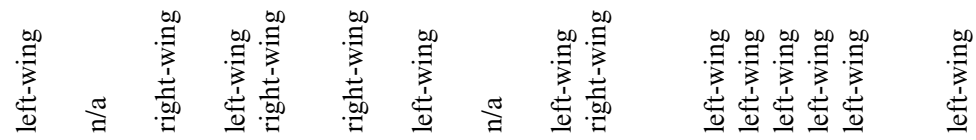

$\stackrel{\varrho}{\cong}$

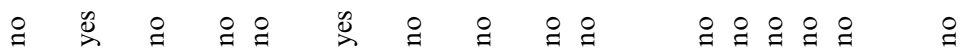

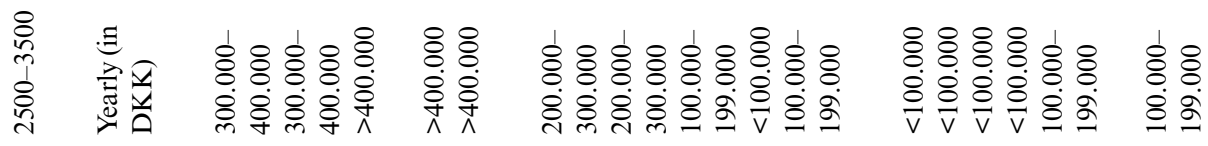

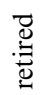

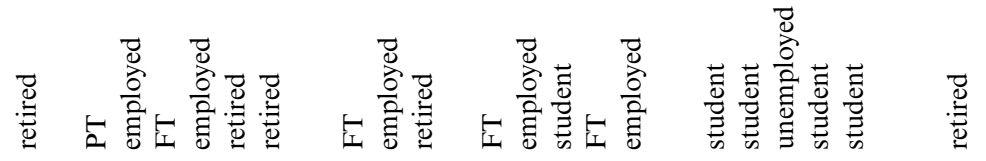

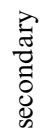

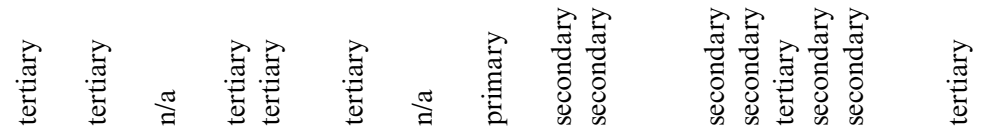

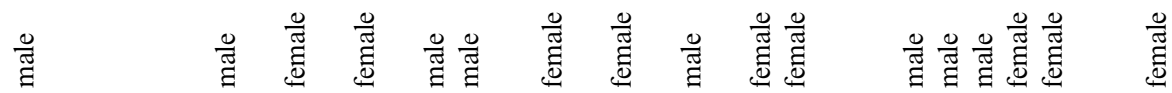

₹

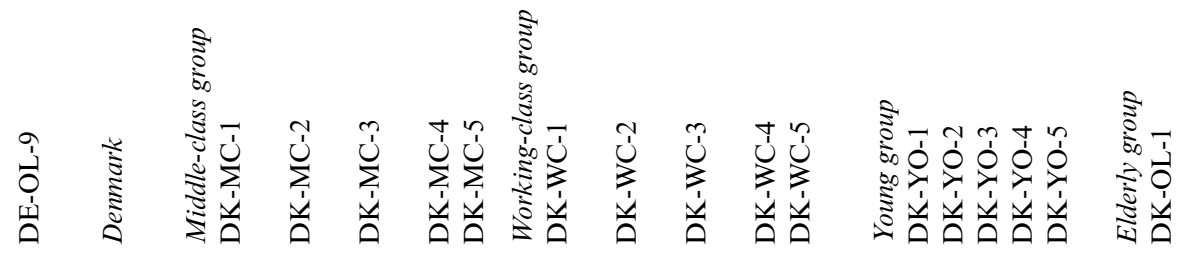




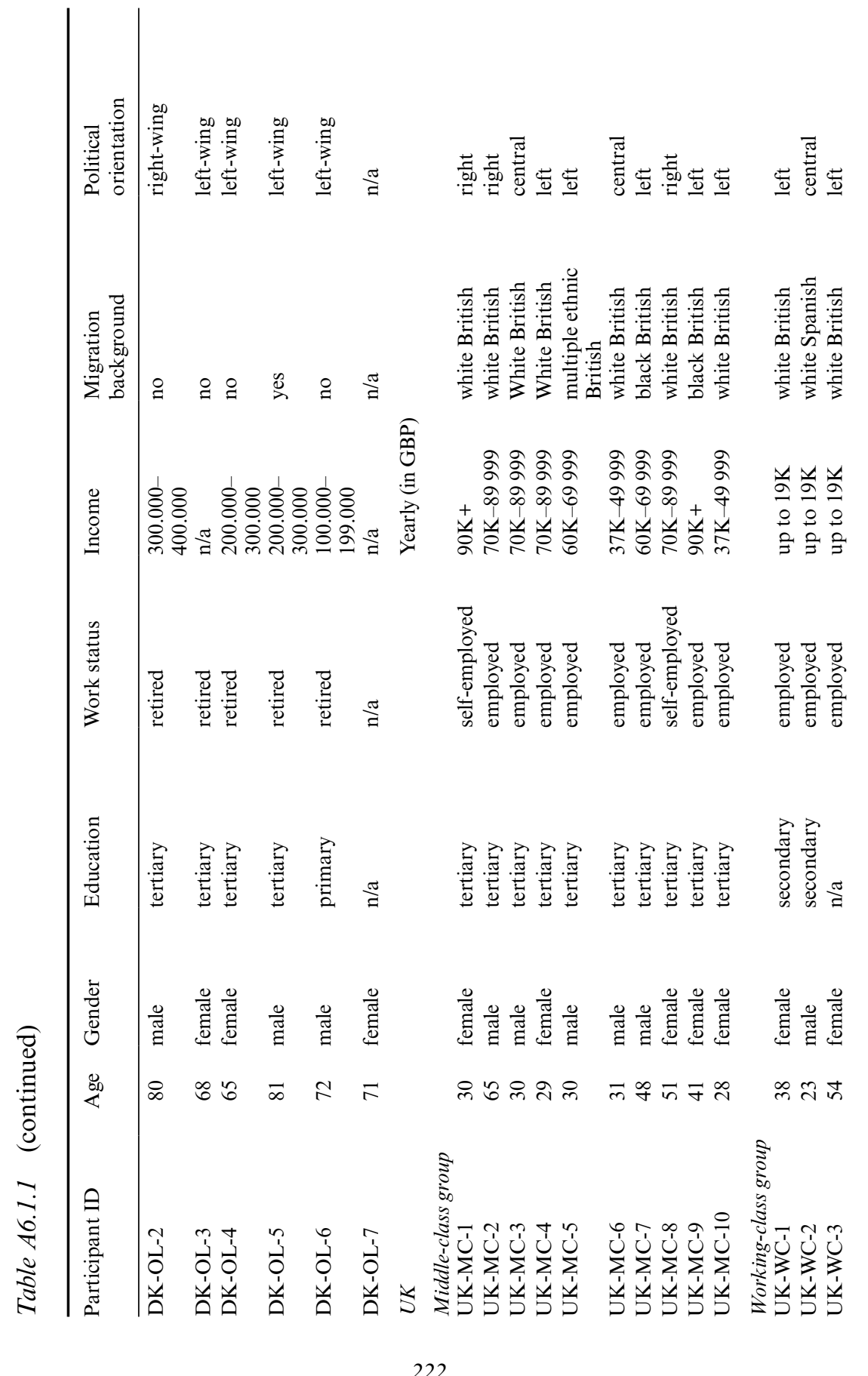




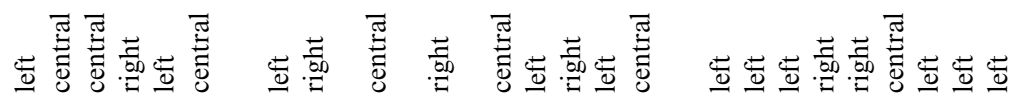

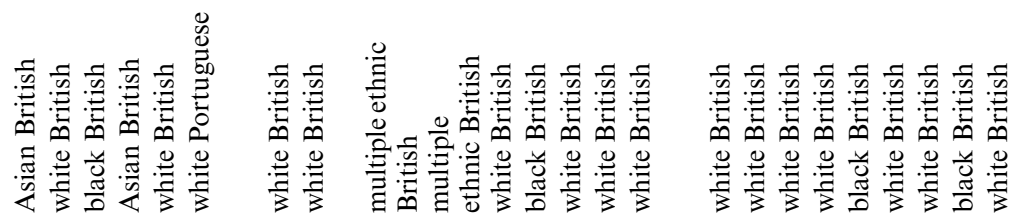

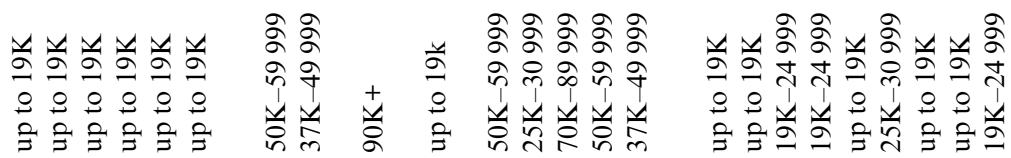

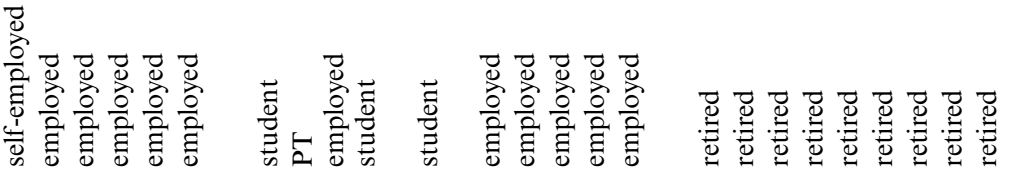

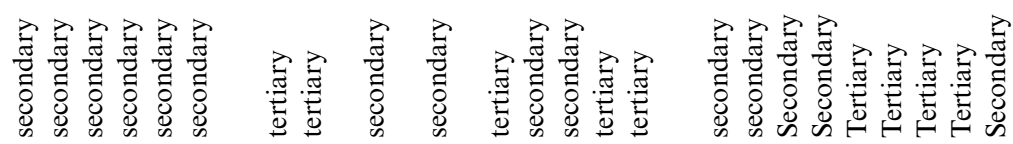

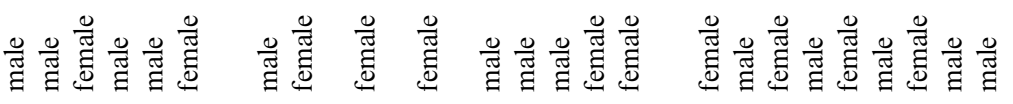

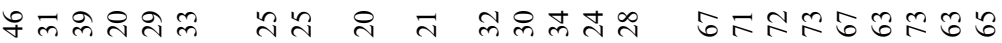

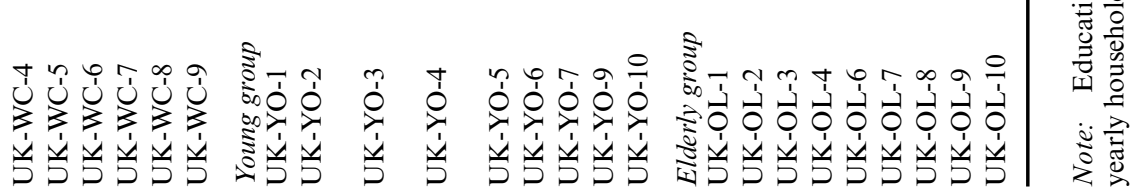




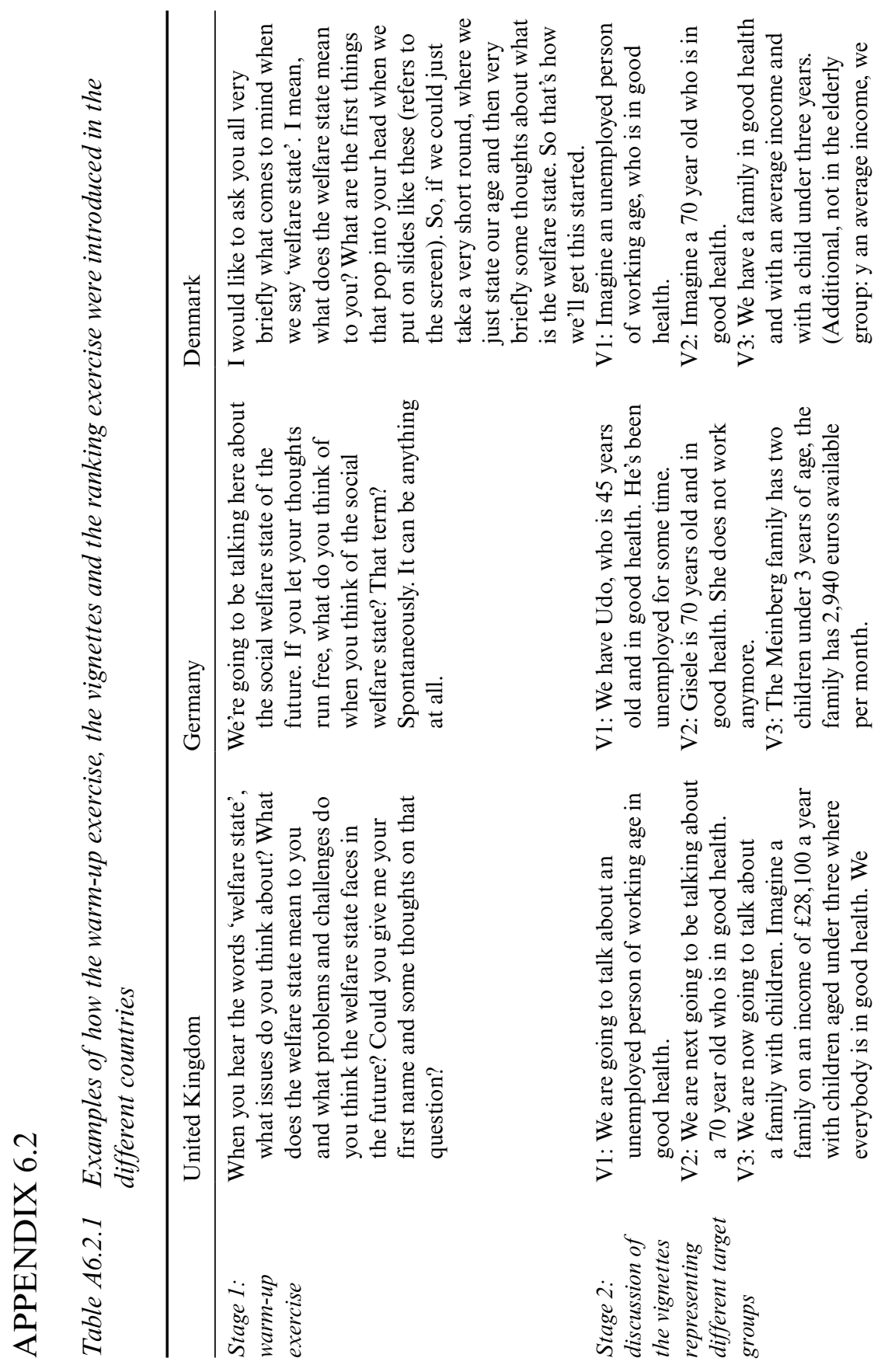




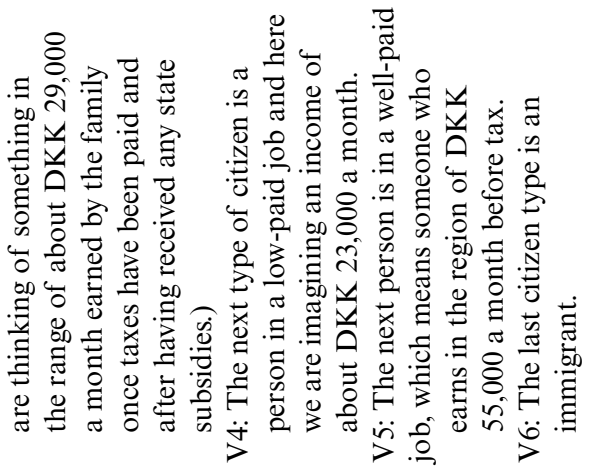

İ

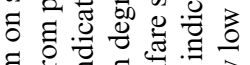
छี Ð

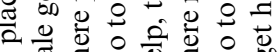

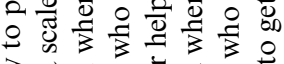

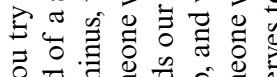

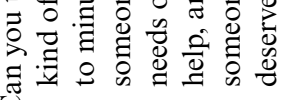

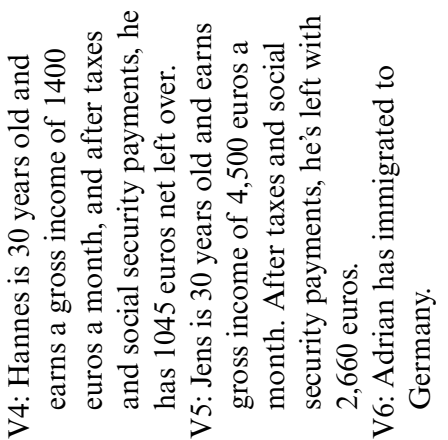

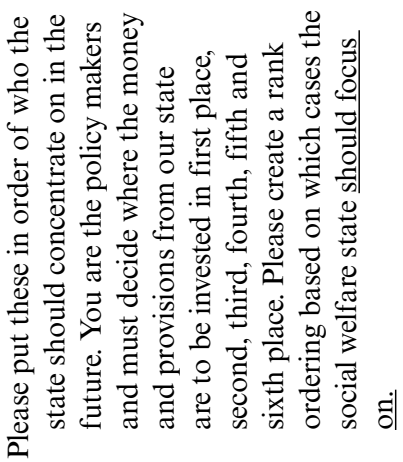

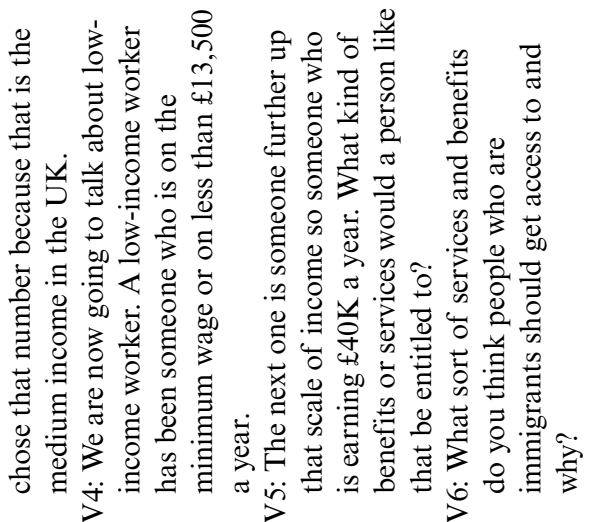

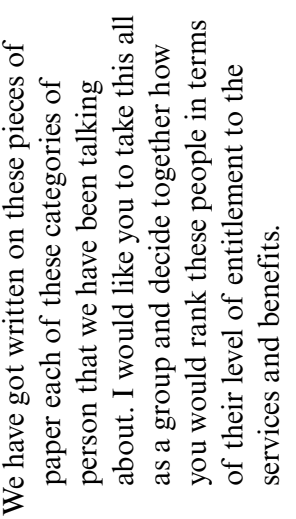

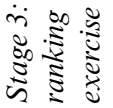




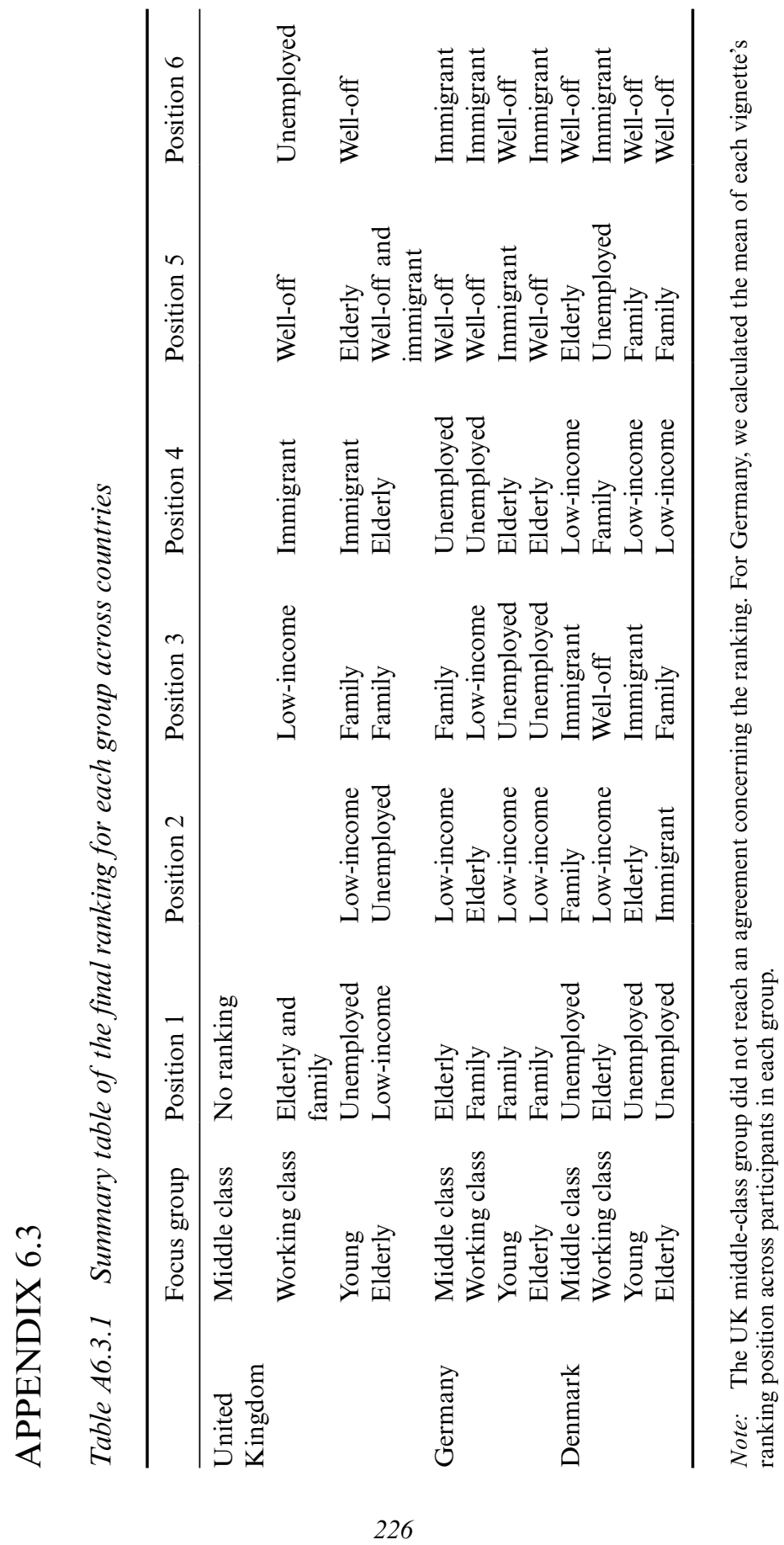




\section{APPENDIX 7.1}

Table A7.1.1 Question wordings and response scales of the dependent and independent variables

\begin{tabular}{|c|c|c|}
\hline Construct & Question wordings & Response scale \\
\hline $\begin{array}{l}\text { Public support for } \\
\text { social welfare }\end{array}$ & $\begin{array}{l}\text { To what extent do you think that society } \\
\text { should grant pensioners/social assistance } \\
\text { recipients/unemployed people the right } \\
\text { to financial support, given that public } \\
\text { funds are limited? }\end{array}$ & $\begin{array}{l}1 \text { (no right) to } 10 \\
\text { (extensive right) }\end{array}$ \\
\hline \multicolumn{3}{|c|}{ Perceived self-interest in benefit $X$} \\
\hline $\begin{array}{l}\text { Likelihood of } \\
\text { receiving benefit } \\
X\end{array}$ & $\begin{array}{l}\text { How likely do you consider it to be that } \\
\text { you will ever receive (early) retirement } \\
\text { benefit/unemployment benefit/social } \\
\text { assistance benefit? }\end{array}$ & $\begin{array}{l}1 \text { (no chance) } \\
\text { to } 7 \text { (currently } \\
\text { receiving the } \\
\text { benefit) }\end{array}$ \\
\hline \multicolumn{3}{|c|}{ Perceived performance of benefit $X$} \\
\hline Benefit fraud & $\begin{array}{l}\text { How often do you think people abuse } \\
\text { pensions/unemployment benefits/social } \\
\text { assistance? }\end{array}$ & $\begin{array}{l}1 \text { (rarely) to } 3 \\
\text { (often) }\end{array}$ \\
\hline Complexity & $\begin{array}{l}\text { How do you feel about the current } \\
\text { system of pensions/unemployment } \\
\text { benefits/social assistance benefits? }\end{array}$ & $\begin{array}{l}1 \text { (complicated) to } \\
5 \text { (simple) }\end{array}$ \\
\hline Efficiency & $\begin{array}{l}\text { How do you feel about the current } \\
\text { system of pensions/unemployment } \\
\text { benefits/social assistance benefits? }\end{array}$ & $\begin{array}{l}1 \text { (poor } \\
\text { implementation) } \\
\text { to } 5 \text { (good } \\
\text { implementation) }\end{array}$ \\
\hline Fairness & $\begin{array}{l}\text { How do you feel about the current } \\
\text { system of pensions/unemployment } \\
\text { benefits/social assistance benefits? }\end{array}$ & $\begin{array}{l}1 \text { (unfair) to } 5 \\
\text { (fair) }\end{array}$ \\
\hline Societal cost & $\begin{array}{l}\text { How do you feel about the current } \\
\text { system of } \\
\text { pensions/unemployment benefits/social } \\
\text { assistance benefits? }\end{array}$ & $\begin{array}{l}1 \text { (costly for } \\
\text { society) to } \\
5 \text { (cheap for } \\
\text { society) }\end{array}$ \\
\hline \multicolumn{3}{|c|}{ Deservingness criteria for benefit $X$} \\
\hline Reciprocity & See Appendix 7.2 & 0 (no); 1 (yes) \\
\hline Need & See Appendix 7.2 & 0 (no); 1 (yes) \\
\hline
\end{tabular}




\section{APPENDIX 7.2}

\section{Table A7.2.1 CFA of deservingness criteria for the Dutch state AOW pension}

\begin{tabular}{|c|c|}
\hline Survey items & Standardized factor loadings \\
\hline \multicolumn{2}{|l|}{ Reciprocity (Cronbach's $\alpha=0.850$ ) } \\
\hline \multicolumn{2}{|l|}{ Should the pension be higher for those elderly } \\
\hline $\begin{array}{l}\text {.. who have paid a higher versus a lower } \\
\text { contribution to the system? }\end{array}$ & 0.889 \\
\hline $\begin{array}{l}\text {... who have a longer versus a shorter work } \\
\text { history before receiving the benefit? }\end{array}$ & 0.897 \\
\hline $\begin{array}{l}\text {... who have had a paid job versus those who } \\
\text { have not had a paid job? }\end{array}$ & 0.888 \\
\hline \multicolumn{2}{|l|}{ Need (Cronbach's $\alpha=0.719$ ) } \\
\hline \multicolumn{2}{|l|}{ Should the pension be lower for those elderly } \\
\hline $\begin{array}{l}\text {.. who have supplementary income versus no } \\
\text { supplementary income? }\end{array}$ & 0.783 \\
\hline $\begin{array}{l}\text {.. who have a partner with income versus a } \\
\text { partner without income? }\end{array}$ & 0.832 \\
\hline $\begin{array}{l}\text {.. who have children with income versus no } \\
\text { children with income? }\end{array}$ & 0.696 \\
\hline $\begin{array}{l}\text {... who have a large amount of savings versus } \\
\text { little or no savings? }\end{array}$ & 0.641 \\
\hline $\begin{array}{l}\ldots \text { who live together with one of their children } \\
\text { versus those who live alone? }\end{array}$ & 0.827 \\
\hline $\begin{array}{l}\ldots \text { who share a household with a sibling versus } \\
\text { those who live alone? }\end{array}$ & 0.813 \\
\hline Goodness of fit indices: & $\begin{aligned} \text { RMSEA } & =0.064 ; \text { CFI }=0.983 ; \\
& \text { TLI }=0.977\end{aligned}$ \\
\hline
\end{tabular}

\section{Table A7.2.2 CFA of deservingness criteria for Dutch unemployment insurance}

Survey items Standardized factor loadings

Reciprocity (Cronbach's $\alpha=0.607$ )

Should unemployment benefit be higher for those

unemployed people

... who became unemployed at an older versus a

younger age?

... who have paid a higher versus a lower contribution to the system?

... who have a longer versus a shorter work history before receiving the benefit?

Need (Cronbach's $\alpha=0.655$ ) 


\section{Table A7.2.2 (continued)}

\begin{tabular}{|c|c|}
\hline Survey items & Standardized factor loadings \\
\hline \multicolumn{2}{|l|}{$\begin{array}{l}\text { Should unemployment benefit be lower for those } \\
\text { unemployed people }\end{array}$} \\
\hline ... who have a smaller versus a larger household? & 0.704 \\
\hline $\begin{array}{l}\ldots \text { who have a partner with income versus a } \\
\text { partner without income? }\end{array}$ & 0.815 \\
\hline $\begin{array}{l}\text {... who have children with income versus no } \\
\text { children with income? }\end{array}$ & 0.713 \\
\hline $\begin{array}{l}\text {... who have a large amount of savings versus } \\
\text { little or no savings? }\end{array}$ & 0.709 \\
\hline $\begin{array}{l}\text {. who have rich parents versus those who do } \\
\text { not have rich parents? }\end{array}$ & 0.624 \\
\hline Goodness of fit indices: & $\begin{aligned} \text { RMSEA } & =0.052 ; \text { CFI }=0.956 ; \text { TLI } \\
& =0.937\end{aligned}$ \\
\hline
\end{tabular}

\section{Table A7.2.3 CFA of deservingness criteria for Dutch social assistance}

\begin{tabular}{|c|c|}
\hline Survey items & Standardized factor loadings \\
\hline \multicolumn{2}{|l|}{ Reciprocity (Cronbach's $\alpha=0.640$ ) } \\
\hline \multicolumn{2}{|l|}{$\begin{array}{l}\text { Should the social assistance benefit be higher for } \\
\text { those people }\end{array}$} \\
\hline $\begin{array}{l}\text {... who previously had a high income versus a } \\
\text { low income? }\end{array}$ & 0.605 \\
\hline ... who are older versus younger? & 0.536 \\
\hline $\begin{array}{l}\text {... who have paid a higher versus a lower } \\
\text { contribution to the system? }\end{array}$ & 0.880 \\
\hline $\begin{array}{l}\ldots \text { who have a longer versus a shorter work } \\
\text { history before receiving the benefit? }\end{array}$ & 0.918 \\
\hline \multicolumn{2}{|l|}{ Need (Cronbach's $\alpha=0.693$ ) } \\
\hline \multicolumn{2}{|l|}{$\begin{array}{l}\text { Should the social assistance benefit be lower for } \\
\text { those people }\end{array}$} \\
\hline $\begin{array}{l}\text {... who have supplementary income versus no } \\
\text { supplementary income? }\end{array}$ & 0.639 \\
\hline ... who have a smaller versus a larger household? & 0.680 \\
\hline $\begin{array}{l}\ldots \text { who have a partner with income versus a } \\
\text { partner without income? }\end{array}$ & 0.914 \\
\hline $\begin{array}{l}\text {... who have children with income versus no } \\
\text { children with income? }\end{array}$ & 0.681 \\
\hline $\begin{array}{l}\text {... who have a large amount of savings versus } \\
\text { little or no savings? }\end{array}$ & 0.624 \\
\hline $\begin{array}{l}\text {... who have a partner versus those who have } \\
\text { no partner? }\end{array}$ & 0.617 \\
\hline Goodness of fit indices: & $\begin{aligned} \text { RMSEA }= & 0.046 ; \text { CFI }=0.960 ; \text { TLI } \\
& =0.948\end{aligned}$ \\
\hline
\end{tabular}




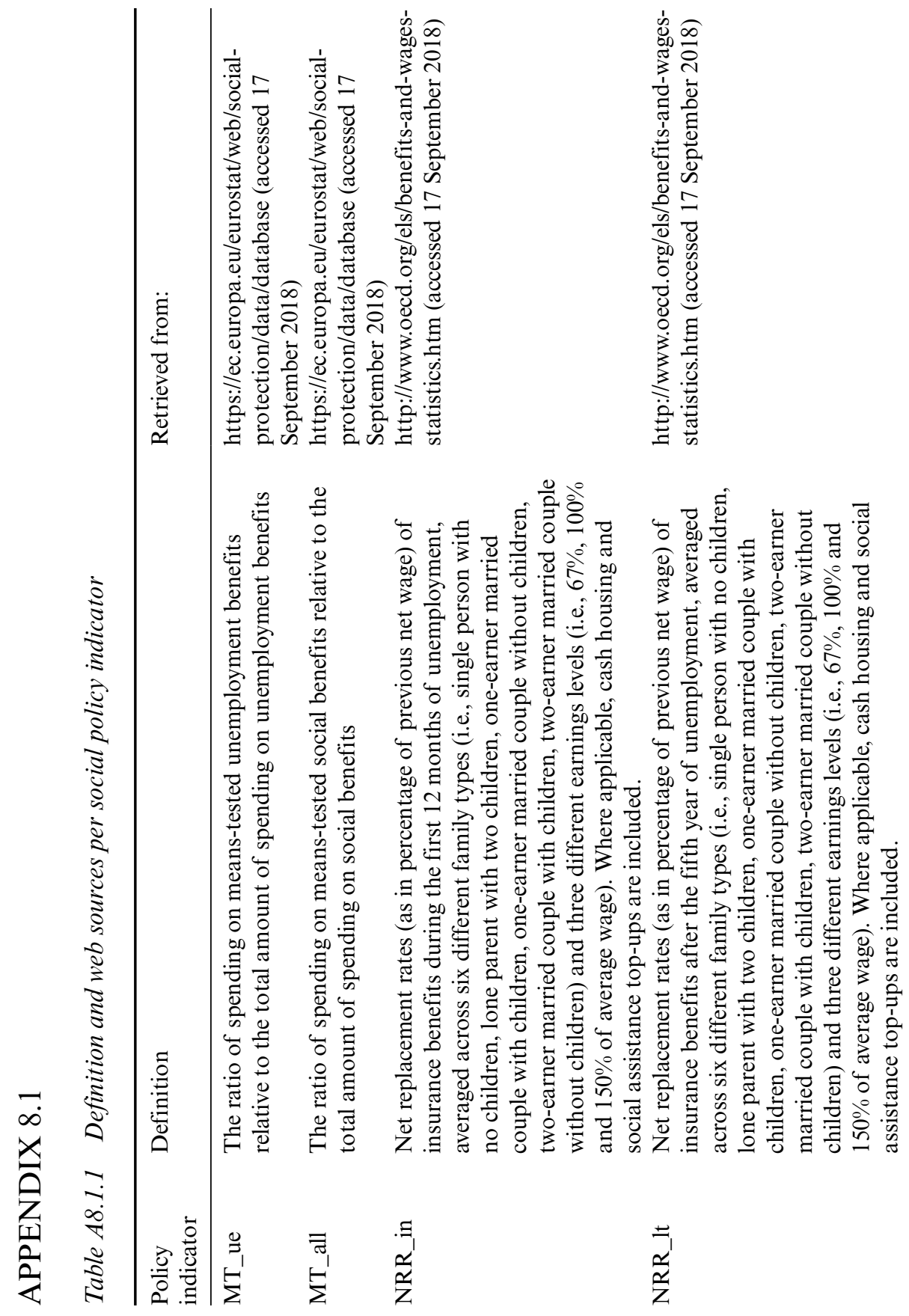




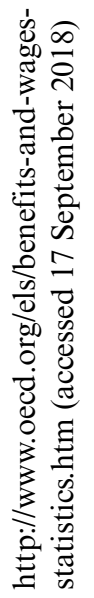

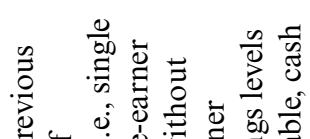

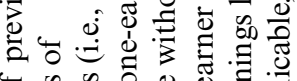
पे कै

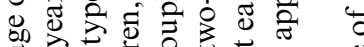

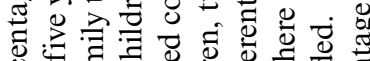

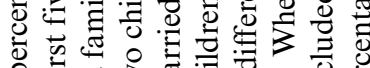

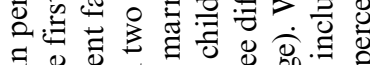

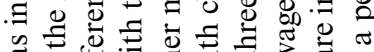
(2)

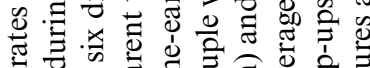
च

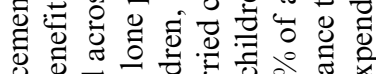
ชั च च : 원

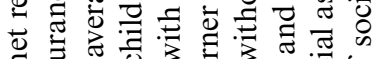

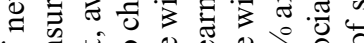

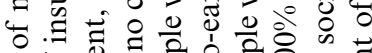

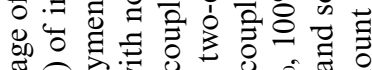
कृ

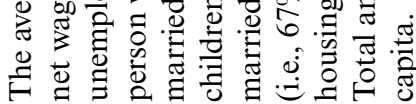
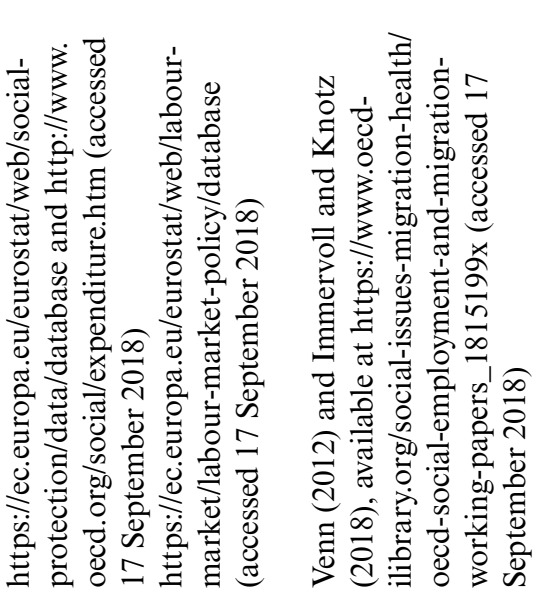
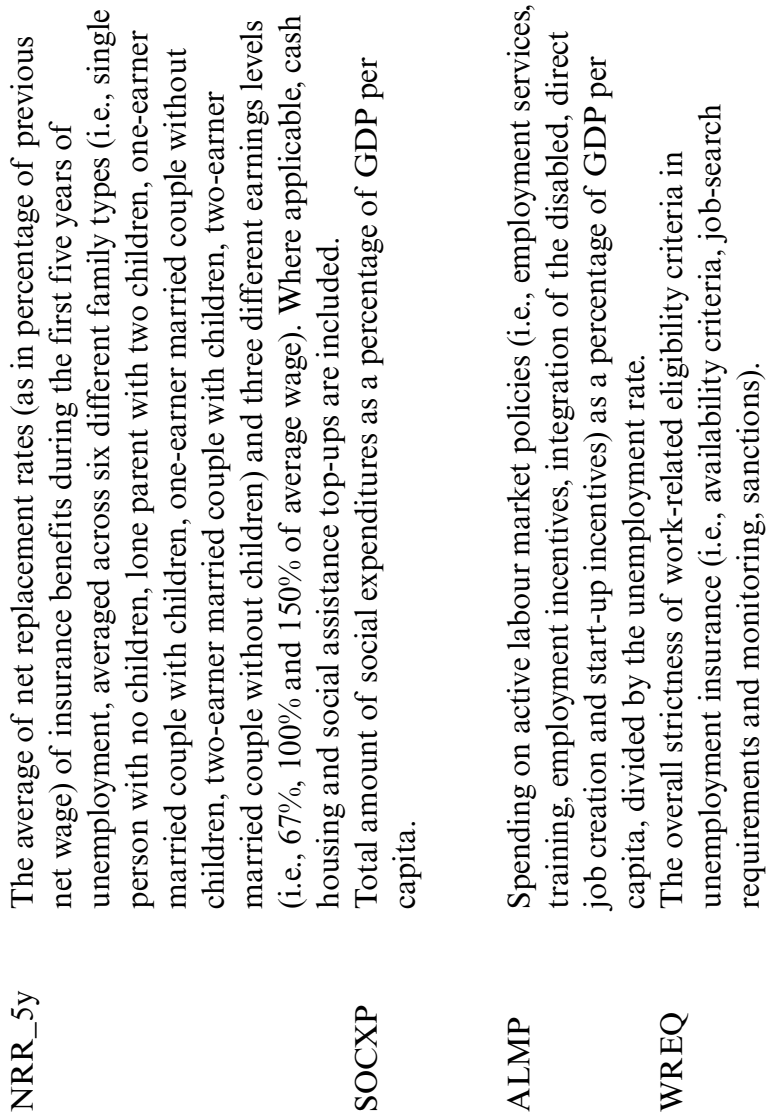
Table A8.1.2 Data matrix of the contextual variables

\begin{tabular}{|c|c|c|c|c|c|c|c|c|}
\hline \multirow[t]{2}{*}{ Country } & \multicolumn{2}{|c|}{ MT_UE } & \multicolumn{2}{|c|}{ MT_all } & \multicolumn{2}{|c|}{ NRR_in } & \multicolumn{2}{|c|}{ NRR_lt } \\
\hline & ‘08 & 15 & '08 & 15 & 08 & 15 & 08 & 15 \\
\hline AT & $\mathrm{Np}$ & 0.245 & $\mathrm{np}$ & $?$ & $\mathrm{np}$ & 68 & $\mathrm{np}$ & 60 \\
\hline $\mathrm{BE}$ & 0 & 0 & 0.051 & 0.049 & 62 & 69 & 60 & 54 \\
\hline BG & 0 & $\mathrm{np}$ & 0.047 & $\mathrm{~Np}$ & 61 & $\mathrm{np}$ & 34 & $\mathrm{np}$ \\
\hline $\mathrm{CH}$ & 0.056 & 0.034 & 0.065 & 0.076 & 83 & 80 & 53 & 52 \\
\hline $\mathrm{CY}$ & 0.005 & $\mathrm{np}$ & 0.122 & $\mathrm{~Np}$ & $\mathrm{md}$ & $\mathrm{np}$ & $\mathrm{md}$ & $\mathrm{np}$ \\
\hline $\mathrm{CZ}$ & 0 & 0 & 0.021 & 0.027 & 67 & 70 & 48 & 48 \\
\hline DE & 0.532 & 0.535 & 0.120 & 0.125 & 74 & 73 & 52 & 48 \\
\hline DK & 0 & $\mathrm{np}$ & 0.032 & $\mathrm{~Np}$ & 76 & $\mathrm{np}$ & 61 & 0 \\
\hline EE & 0 & 0 & 0.004 & 0.005 & 62 & 65 & 33 & 41 \\
\hline ES & 0.182 & 0.305 & 0.136 & 0.134 & 69 & 66 & 35 & 34 \\
\hline FI & 0.187 & 0.297 & 0.042 & 0.062 & 69 & 75 & 57 & 61 \\
\hline FR & 0.103 & 0.073 & 0.142 & 0.109 & 72 & 72 & 46 & 46 \\
\hline GB & 0.267 & 0.325 & 0.146 & 0.119 & 53 & 54 & 52 & 52 \\
\hline GR & 0.002 & $\mathrm{np}$ & 0.075 & $\mathrm{np}$ & 49 & $\mathrm{np}$ & 16 & $\mathrm{np}$ \\
\hline HR & md & $\mathrm{np}$ & $\mathrm{md}$ & $\mathrm{np}$ & $\mathrm{md}$ & $\mathrm{np}$ & $\mathrm{md}$ & $\mathrm{np}$ \\
\hline $\mathrm{HU}$ & 0.304 & 0.495 & 0.054 & 0.042 & 69 & 58 & 47 & 28 \\
\hline IE & 0.428 & 0.775 & 0.253 & 0.302 & 69 & 66 & 67 & 64 \\
\hline IL & md & md & $\mathrm{md}$ & md & 71 & 84 & 38 & 39 \\
\hline IS & $\mathrm{np}$ & 0 & $\mathrm{np}$ & 0.230 & $\mathrm{np}$ & 68 & np & 56 \\
\hline IT & $\mathrm{np}$ & 0 & $\mathrm{np}$ & 0.077 & $\mathrm{np}$ & 71 & $\mathrm{np}$ & 17 \\
\hline LT & $\mathrm{np}$ & 0 & $\mathrm{np}$ & 0.032 & np & 66 & np & $\mathrm{md}$ \\
\hline LV & 0 & $\mathrm{np}$ & 0.020 & $\mathrm{np}$ & 83 & $\mathrm{np}$ & 43 & $\mathrm{np}$ \\
\hline NL & 0.365 & 0.263 & 0.145 & 0.145 & 75 & 74 & 58 & 55 \\
\hline NO & 0 & 0 & 0.044 & 0.036 & 70 & 69 & 53 & 52 \\
\hline PL & 0.124 & 0.193 & 0.043 & 0.038 & 55 & 57 & 43 & 42 \\
\hline PT & 0.181 & 0.136 & 0.099 & 0.080 & 84 & 78 & 40 & 33 \\
\hline RO & 1 & $\mathrm{np}$ & 0.048 & $\mathrm{np}$ & 53 & $\mathrm{np}$ & 26 & $\mathrm{np}$ \\
\hline RU & $\mathrm{md}$ & $\mathrm{md}$ & md & md & $\mathrm{md}$ & $\mathrm{md}$ & $\mathrm{md}$ & $\mathrm{md}$ \\
\hline SE & 0 & 0 & 0.027 & 0.026 & 65 & 60 & 52 & 50 \\
\hline SI & 0.002 & 0 & 0.085 & 0.078 & 74 & 75 & 57 & 56 \\
\hline SK & 0.006 & $\mathrm{np}$ & 0.049 & $\mathrm{np}$ & 72 & $\mathrm{np}$ & 35 & $\mathrm{np}$ \\
\hline TR & 0.176 & $\mathrm{np}$ & md & np & 52 & $\mathrm{np}$ & 14 & $\mathrm{np}$ \\
\hline UA & $\mathrm{md}$ & $\mathrm{np}$ & $\mathrm{md}$ & np & $\mathrm{md}$ & $\mathrm{np}$ & $\mathrm{md}$ & $\mathrm{np}$ \\
\hline
\end{tabular}

Note: $\mathrm{np}=$ not participating in the ESS wave. $\mathrm{md}=$ missing data due to data unavailability. From the data matrix, one can read which countries were excluded in the multilevel models introducing contextual variables because of missing data. Russia and Ukraine, for example, were excluded altogether because of missing data across the board, but Cyprus, as a second example, was only excluded from the models including net replacement rates. 


\begin{tabular}{|c|c|c|c|c|c|c|c|c|c|c|c|c|}
\hline \multirow[t]{2}{*}{ Country } & \multicolumn{2}{|c|}{ NRR_5y } & \multicolumn{2}{|c|}{ SOCXP } & \multicolumn{2}{|c|}{ ALMP } & \multicolumn{2}{|c|}{ WREQ } & \multicolumn{2}{|c|}{ GDP } & \multicolumn{2}{|l|}{ UE } \\
\hline & ‘08 & 15 & '08 & 15 & '08 & 16 & 11 & 17 & 08 & 16 & '08 & 16 \\
\hline AT & $\mathrm{np}$ & 69 & $\mathrm{np}$ & 27.8 & $\mathrm{np}$ & 0.097 & $\mathrm{np}$ & 2.91 & $\mathrm{np}$ & $?$ & $\mathrm{np}$ & 6.0 \\
\hline $\mathrm{BE}$ & 64 & 63 & 27.7 & 30.3 & 0.072 & 0.067 & 2.39 & 2.98 & 115 & 118 & 7.0 & 7.8 \\
\hline BG & 40 & $\mathrm{np}$ & 14.7 & $\mathrm{np}$ & 0.043 & $\mathrm{np}$ & 2.96 & $\mathrm{np}$ & 44 & $\mathrm{np}$ & 5.6 & $\mathrm{np}$ \\
\hline $\mathrm{CH}$ & 72 & 71 & 23.2 & 27.3 & md & md & 3.55 & 3.55 & 143 & 161 & 3.4 & 4.9 \\
\hline CY & $\mathrm{md}$ & $\mathrm{np}$ & 17.6 & $\mathrm{np}$ & 0.028 & $\mathrm{np}$ & 1.84 & $\mathrm{np}$ & 97 & $\mathrm{np}$ & 3.6 & $\mathrm{np}$ \\
\hline $\mathrm{CZ}$ & 56 & 57 & 17.9 & 19 & 0.025 & 0.061 & 2.69 & 2.71 & 81 & 88 & 4.4 & 4.0 \\
\hline $\mathrm{DE}$ & 65 & 57 & 27.1 & 29.1 & 0.075 & 0.064 & 3.14 & 3.14 & 116 & 124 & 7.3 & 4.1 \\
\hline DK & 76 & $\mathrm{np}$ & 28.9 & $\mathrm{np}$ & 0.284 & $\mathrm{np}$ & 3.45 & $\mathrm{np}$ & 123 & $\mathrm{np}$ & 3.3 & $\mathrm{np}$ \\
\hline EE & 35 & 48 & 14.7 & 16.4 & 0.005 & 0.026 & 4.19 & 4.19 & 68 & 75 & 5.5 & 6.8 \\
\hline ES & 47 & 43 & 21.4 & 24.6 & 0.052 & 0.022 & 2.70 & 2.70 & 103 & 92 & 11.3 & 19.6 \\
\hline FI & 70 & 72 & 25.1 & 31.6 & 0.100 & 0.095 & 2.76 & 2.79 & 118 & 109 & 6.4 & 8.8 \\
\hline FR & 60 & 59 & 33.9 & 30.4 & 0.077 & 0.070 & 3.19 & 3.3 & 107 & 104 & 7.8 & 10.1 \\
\hline GB & 61 & 60 & 25.8 & 28.6 & 0.006 & $\mathrm{md}$ & 3.29 & 3.65 & 115 & 108 & 5.6 & 4.8 \\
\hline GR & 21 & np & 26.0 & $\mathrm{np}$ & 0.018 & $\mathrm{np}$ & 2.73 & $\mathrm{np}$ & 94 & $\mathrm{np}$ & 7.7 & $\mathrm{np}$ \\
\hline HR & $\mathrm{md}$ & np & 18.8 & np & md & $\mathrm{np}$ & 3.80 & $\mathrm{np}$ & 64 & $\mathrm{np}$ & 8.4 & $\mathrm{np}$ \\
\hline $\mathrm{HU}$ & 53 & 24 & 22.3 & 20.0 & 0.033 & 0.170 & 2.05 & 2.47 & 64 & 67 & 7.8 & 5.1 \\
\hline IE & 80 & 74 & 20.7 & 16.3 & 0.083 & 0.047 & 2.97 & 2.71 & 133 & 183 & 6.3 & 8.4 \\
\hline IL & 43 & 47 & 15.4 & 16.1 & md & md & 2.39 & $\mathrm{md}$ & $\mathrm{md}$ & $\mathrm{md}$ & 6.1 & 4.8 \\
\hline IS & np & 64 & $\mathrm{np}$ & 22.8 & np & $\mathrm{md}$ & $\mathrm{np}$ & 3.08 & $\mathrm{np}$ & 128 & $\mathrm{np}$ & 8.4 \\
\hline IT & $\mathrm{np}$ & 23 & $\mathrm{np}$ & 29.9 & $\mathrm{np}$ & 0.035 & $\mathrm{np}$ & 3.31 & $\mathrm{np}$ & 97 & $\mathrm{np}$ & 11.7 \\
\hline LT & np & 37 & np & 15.6 & $\mathrm{np}$ & 0.030 & $\mathrm{np}$ & 3.06 & np & 75 & np & 7.9 \\
\hline LV & 48 & np & 12.1 & $\mathrm{np}$ & 0.010 & $\mathrm{np}$ & 3.13 & $\mathrm{np}$ & 56 & $\mathrm{np}$ & 7.5 & $\mathrm{np}$ \\
\hline NL & 73 & 70 & 26.4 & 30.2 & 0.216 & 0.080 & 3.43 & 3.09 & 134 & 128 & 3.1 & 6.0 \\
\hline NO & 69 & 69 & 21.8 & 27.9 & 0.162 & 0.080 & 3.05 & 3.37 & 189 & 148 & 2.5 & 4.7 \\
\hline PL & 50 & 48 & 19.3 & 19.1 & 0.065 & 0.059 & 3.10 & 3.10 & 56 & 68 & 7.1 & 6.2 \\
\hline PT & 61 & 49 & 23.4 & 25.7 & 0.051 & 0.037 & 4.15 & 3.89 & 78 & 77 & 7.7 & 11.1 \\
\hline RO & 26 & $\mathrm{np}$ & 13.7 & $\mathrm{np}$ & 0.010 & $\mathrm{np}$ & 3.40 & $\mathrm{np}$ & 47 & $\mathrm{np}$ & 5.8 & $\mathrm{np}$ \\
\hline RU & $\mathrm{md}$ & md & $\mathrm{md}$ & md & $\mathrm{md}$ & $\mathrm{md}$ & $\mathrm{md}$ & md & $\mathrm{md}$ & $\mathrm{md}$ & $\mathrm{md}$ & $\mathrm{md}$ \\
\hline SE & 67 & 62 & 27.7 & 29.2 & 0.102 & 0.128 & 3.37 & 3.37 & 122 & 123 & 6.2 & 7.0 \\
\hline SI & 67 & 65 & 21.0 & 23.8 & 0.021 & 0.020 & 4.06 & 4.06 & 91 & 83 & 4.4 & 8.0 \\
\hline SK & 37 & $\mathrm{np}$ & 15.7 & $\mathrm{np}$ & md & $\mathrm{np}$ & 3.38 & $\mathrm{np}$ & 75 & $\mathrm{np}$ & 9.5 & $\mathrm{np}$ \\
\hline TR & 8 & $\mathrm{np}$ & 11.4 & 12.0 & $\mathrm{md}$ & $\mathrm{np}$ & 2.41 & 2.41 & 47 & 64 & 9.7 & 10.8 \\
\hline UA & $\mathrm{md}$ & $\mathrm{np}$ & $\mathrm{md}$ & $\mathrm{np}$ & $\mathrm{md}$ & $\mathrm{np}$ & $\mathrm{md}$ & $\mathrm{np}$ & $\mathrm{md}$ & $\mathrm{np}$ & $\mathrm{md}$ & $\mathrm{np}$ \\
\hline
\end{tabular}




\section{APPENDIX 8.2}

\section{Table A8.2.1 The impact of the individual-level control variables on popular deservingness perceptions}

Need (low-high) Job-seeking

efforts

(low-high)

\begin{tabular}{|c|c|c|}
\hline $\begin{array}{l}\text { Individual level } \\
\text { ESS round (ref. }=2008 / 09 \text { ) }\end{array}$ & & \\
\hline 2016/17 & $-0.076 *$ & -0.048 \\
\hline Age (in years) & $0.054 * * *$ & $-0.086 * * *$ \\
\hline Gender $($ ref. $=$ male $)$ & & \\
\hline Female & -0.002 & $-0.015 *$ \\
\hline Education (in years) & $0.139 * * *$ & $-0.019 *$ \\
\hline Current work status (ref. = paid work) & & \\
\hline in education & $0.045 * * *$ & $-0.016^{*}$ \\
\hline unemployed/disabled & $0.053 * * *$ & $-0.043 * * *$ \\
\hline retired & $-0.025 * *$ & -0.010 \\
\hline other (including housework) & 0.004 & $-0.017 * *$ \\
\hline $\begin{array}{l}\text { Past experience of unemployment for more than } 3 \text { months } \\
\text { (ref. = no) }\end{array}$ & $0.036 * * *$ & $0.051 * * *$ \\
\hline Predicted likelihood of future unemployment & $0.015^{*}$ & $-0.017 *$ \\
\hline $\begin{array}{l}\text { Subjective income (ref. = living comfortably on present } \\
\text { income) }\end{array}$ & & \\
\hline Coping & $-0.038 * * *$ & $-0.024 * *$ \\
\hline Difficult & -0.018 & $-0.073 * * *$ \\
\hline Very difficult & $0.036 * * *$ & $-0.112 * * *$ \\
\hline Left-right ideology (ref. = centre) & & \\
\hline Left-wing & $0.087 * * *$ & $-0.050 * * *$ \\
\hline Right-wing & -0.012 & $0.043 * * *$ \\
\hline Missing & 0.010 & $-0.024 * *$ \\
\hline Egalitarianism & $0.061 * * *$ & $-0.087 * * *$ \\
\hline Country-year level & & \\
\hline Net replacement rate, long-term unemployment & $-0.272 * *$ & $-0.313 * *$ \\
\hline Total social expenditures & $-0.200 * *$ & \\
\hline Spending on active labour market policies & & $0.330 *$ \\
\hline Strictness of work-related eligibility criteria & 0.095 & \\
\hline GDP per capita (in PPS per head) & $-0.525 * * *$ & $0.535 * * *$ \\
\hline Unemployment rate & $0.193 * *$ & 0.219 \\
\hline $\mathrm{R}^{2}$ individual level & 0.062 & 0.042 \\
\hline $\mathrm{N}$ individual level & 76197 & 67167 \\
\hline $\mathrm{R}^{2}$ country-year level & 0.841 & 0.375 \\
\hline $\mathrm{N}$ country-year level & 43 & 37 \\
\hline
\end{tabular}

Note: $* \mathrm{p}<0.05, * * \mathrm{p}<0.01, * * * \mathrm{p}<0.001$. Data were weighted using the poststratification weights provided by the ESS. The reported parameters are standardized regression coefficients. 
Table A8.2.2 The impact of the individual-level control variables on public support for unemployment provision

\begin{tabular}{|c|c|c|}
\hline \multicolumn{3}{|l|}{ Fixed effects } \\
\hline Intercept & 6.758 & $* * *$ \\
\hline \multicolumn{3}{|l|}{ ESS round (ref. $=2008 / 09)$} \\
\hline $2016 / 17$ & -0.107 & \\
\hline \multicolumn{3}{|l|}{ Deservingness perceptions } \\
\hline need & 0.175 & $* * *$ \\
\hline job-seeking efforts & 0.309 & $* * *$ \\
\hline \multicolumn{3}{|l|}{ Interaction terms } \\
\hline need*ESS round 2016/17 & 0.019 & \\
\hline job-seeking efforts*ESS round 2016/17 & -0.084 & $*$ \\
\hline Age (in years) & 0.001 & \\
\hline \multicolumn{3}{|l|}{ Gender $($ ref. $=$ male $)$} \\
\hline Female & 0.077 & $* *$ \\
\hline Education (in years) & -0.023 & $* * *$ \\
\hline \multicolumn{3}{|l|}{ Current work status (ref. = paid work) } \\
\hline in education & 0.082 & \\
\hline unemployed/disabled & 0.217 & *** \\
\hline retired & 0.136 & $* * *$ \\
\hline other (including housework) & 0.102 & $* *$ \\
\hline \multicolumn{3}{|c|}{ Past experience of unemployment for more than 3 months } \\
\hline Predicted likelihood of future unemployment & -0.015 & \\
\hline \multicolumn{3}{|c|}{ Subjective income (ref. = living comfortably on present income) } \\
\hline coping & 0.021 & $* * *$ \\
\hline difficult & 0.177 & $* * *$ \\
\hline very difficult & 0.311 & \\
\hline \multicolumn{3}{|l|}{ Left-right ideology (ref. $=$ centre $)$} \\
\hline left-wing & 0.161 & $* * *$ \\
\hline right-wing & -0.050 & \\
\hline missing & 0.166 & $* * *$ \\
\hline egalitarianism & 0.332 & $* * *$ \\
\hline \multicolumn{3}{|l|}{ Random effects } \\
\hline \multicolumn{3}{|l|}{ Random slope variances } \\
\hline job-seeking efforts & 0.012 & $* * *$ \\
\hline Residual variance (individual level) & 4.184 & $* * *$ \\
\hline Random intercept variance (country-year level) & 0.261 & $* * *$ \\
\hline $\mathrm{N}$ individual level & 89974 & \\
\hline $\mathrm{N}$ country-year level & 52 & \\
\hline
\end{tabular}

Note: ${ }^{*} \mathrm{p}<0.05,{ }^{* *} \mathrm{p}<0.01,{ }^{* * *} \mathrm{p}<0.001$. Data were weighted using the poststratification weights provided by the ESS. The reported parameters are unstandardized regression coefficients. 


\section{APPENDIX 8.3}

Table A8.3.1 Country-specific standardized effects of deservingness perceptions on public support for unemployment provision

\begin{tabular}{|c|c|c|c|c|}
\hline \multirow[t]{2}{*}{ Country } & \multicolumn{2}{|c|}{ ESS 2008} & \multicolumn{2}{|c|}{ ESS 2016} \\
\hline & Job-seeking efforts & Need & Job-seeking efforts & Need \\
\hline AT & & & $0.204 * * *$ & -0.014 \\
\hline $\mathrm{BE}$ & $0.179 * * *$ & $0.083^{* *}$ & $-0.283^{* * *}$ & $0.065^{* *}$ \\
\hline BG & $0.163 * * *$ & $0.142^{* * *}$ & & \\
\hline $\mathrm{CH}$ & $0.161 * * *$ & $0.086^{* * *}$ & $0.115^{* * *}$ & $0.075^{* *}$ \\
\hline CY & 0.014 & $-0.121 * * *$ & & \\
\hline $\mathrm{CZ}$ & $0.180 * * *$ & $0.126^{* * *}$ & $0.223^{* * *}$ & $0.278 * * *$ \\
\hline $\mathrm{DE}$ & $0.067 * *$ & $0.172 * * *$ & $0.228 * * *$ & $0.060 * *$ \\
\hline DK & $0.246^{* * *}$ & 0.009 & & \\
\hline $\mathrm{EE}$ & $0.134 * * *$ & $0.125 * * *$ & $0.150 * * *$ & $0.162 * * *$ \\
\hline ES & $0.078 * * *$ & $0.140 * * *$ & $0.169^{* * *}$ & $0.209 * * *$ \\
\hline FI & $0.179 * * *$ & $0.057 * *$ & $0.177 * * *$ & $0.088 * * *$ \\
\hline FR & $0.248 * * *$ & $0.188 * * *$ & $0.219 * * *$ & $0.173 * * *$ \\
\hline GB & $0.205^{* * *}$ & $0.185^{* * *}$ & $0.223 * * *$ & $0.150 * * *$ \\
\hline GR & $0.181 * * *$ & $0.133^{* * *}$ & & \\
\hline HR & $0.163^{* * *}$ & $0.214 * * *$ & & \\
\hline HU & $0.084 * *$ & $0.268 * * *$ & $0.180 * * *$ & $0.121 * * *$ \\
\hline IE & $0.167 * * *$ & $0.139 * * *$ & $0.224 * * *$ & $0.081 * * *$ \\
\hline IL & $0.137 * * *$ & $0.075^{* * *}$ & $0.062 * *$ & $0.068 * *$ \\
\hline IS & & & $0.177 * * *$ & $0.135^{* *}$ \\
\hline IT & & & $0.231 * * *$ & $0.248 * * *$ \\
\hline LT & & & $0.236 * * *$ & $0.217 * * *$ \\
\hline LV & $0.162 * * *$ & $0.151 * * *$ & & \\
\hline NL & $0.219 * * *$ & 0.045 & $0.188 * * *$ & $0.133 * * *$ \\
\hline NO & $0.171 * * *$ & 0.023 & $0.173 * * *$ & 0.028 \\
\hline PL & $0.092 * * *$ & $0.122 * * *$ & $0.183 * * *$ & $0.145^{* * *}$ \\
\hline PT & $0.092 * * *$ & $0.175^{* * *}$ & $0.082 * *$ & $0.272 * * *$ \\
\hline RO & $0.150 * * *$ & $0.237 * * *$ & & \\
\hline RU & $0.188 * * *$ & $0.158 * * *$ & $0.239 * * *$ & $0.244 * * *$ \\
\hline SE & $0.110 * * *$ & $0.084 * * *$ & $0.215^{* * *}$ & 0.019 \\
\hline SI & $0.119 * * *$ & $0.175^{* * *}$ & $0.150 * * *$ & $0.215^{* * *}$ \\
\hline SK & $0.117 * * *$ & $0.151 * * *$ & & \\
\hline TR & 0.034 & $0.271 * * *$ & & \\
\hline UA & $0.193 * * *$ & $0.189 * * *$ & & \\
\hline
\end{tabular}

Note: ${ }^{*} \mathrm{p}<0.05, * * \mathrm{p}<0.01,{ }^{* * *} \mathrm{p}<0.001$. Data were weighted using the poststratification weights provided by the ESS. Reported parameters are standardized regression coefficients, controlled for age, gender, education, work status, subjective income, past experience of unemployment, perceived unemployment risk, egalitarianism and political ideology. Cyprus is a clear outlier because need perceptions have a negative effect on support for social welfare, meaning that Cypriots are more inclined to share welfare resources with unemployed people who they perceive as well-off than with unemployed people who they perceive as poor. 


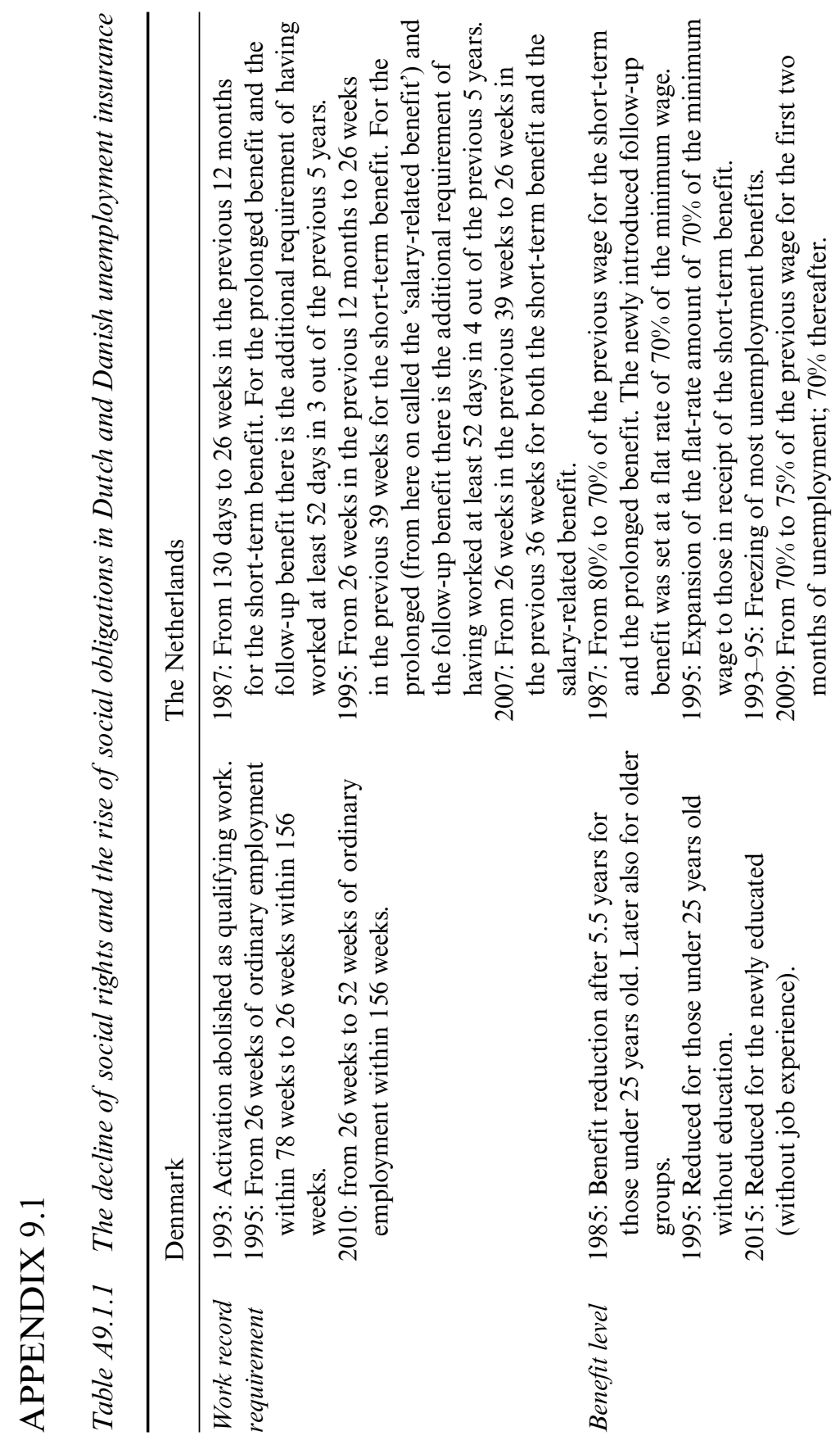




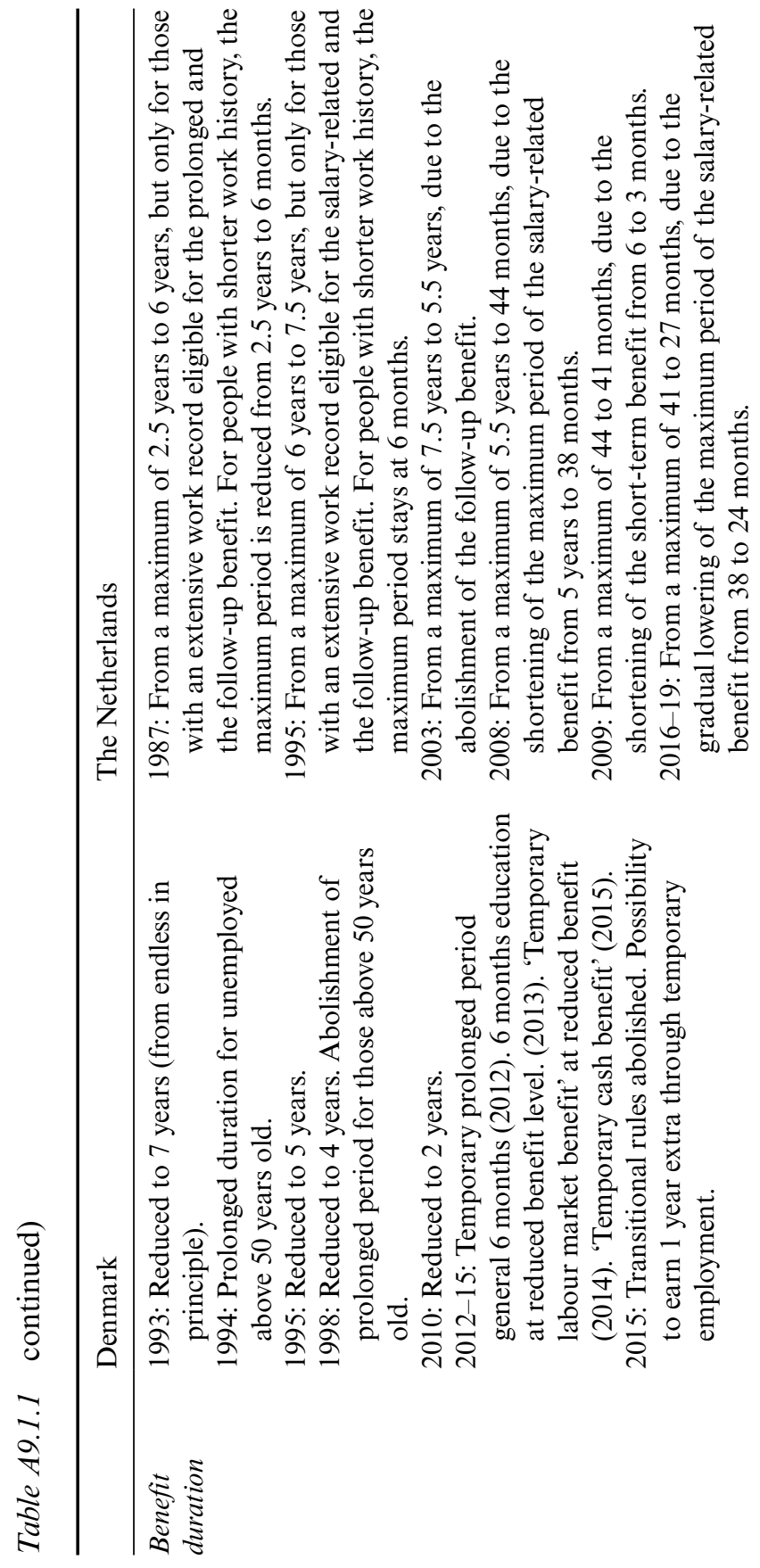




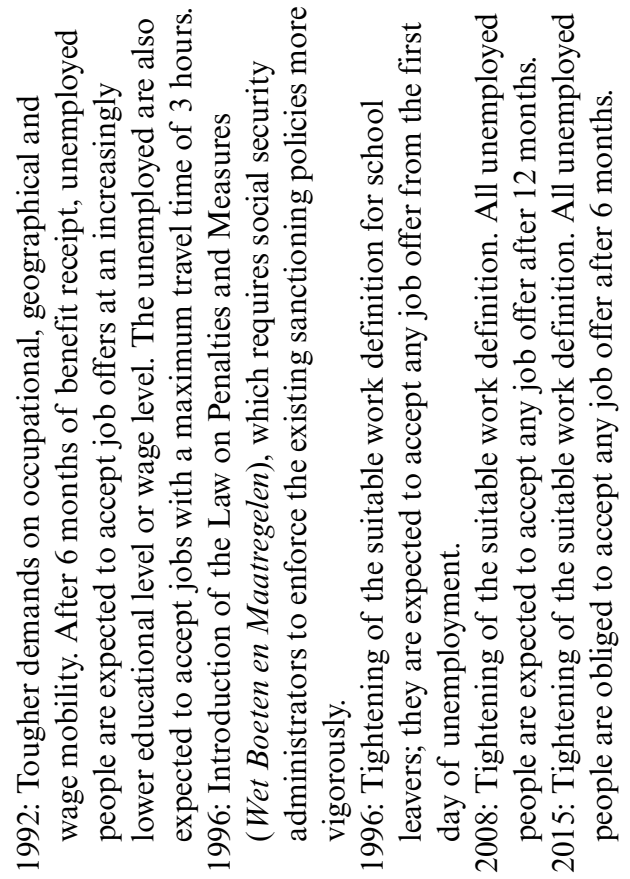

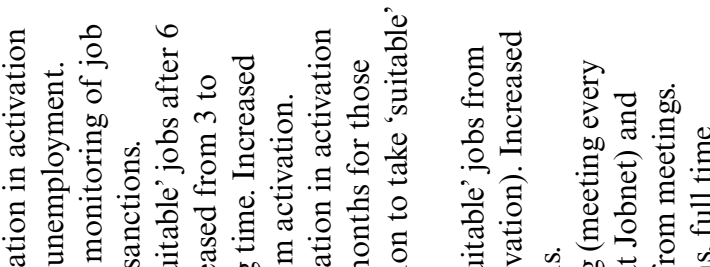

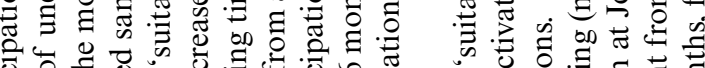

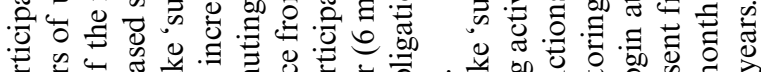

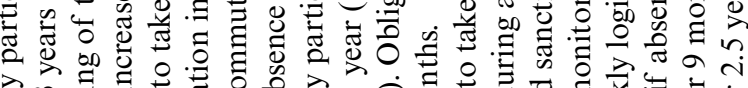

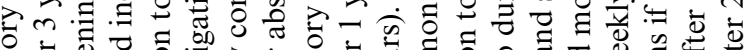

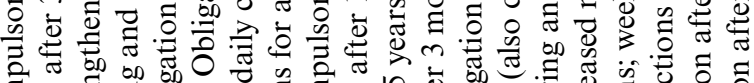

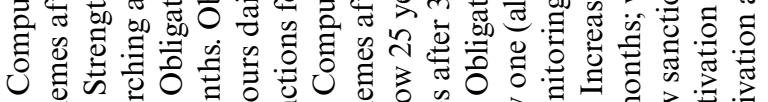

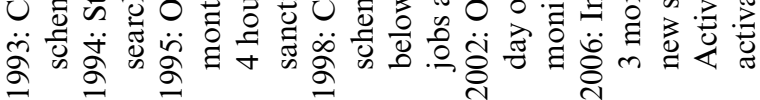

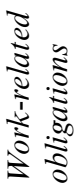

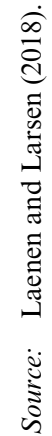




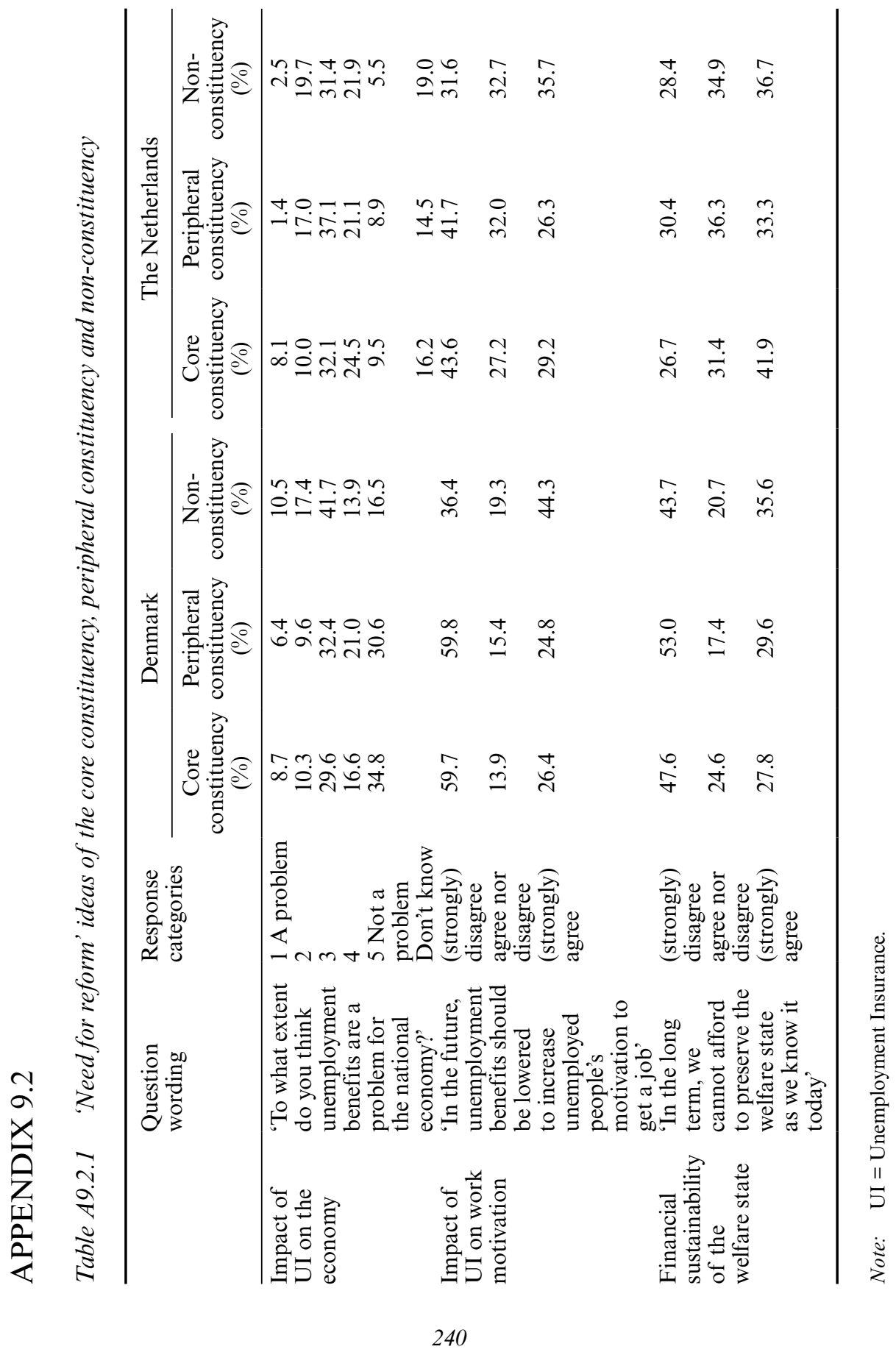




\section{APPENDIX 9.3}

Table A9.3.1 Principal component factor analysis of the deservingness items

\begin{tabular}{|c|c|c|}
\hline \multicolumn{2}{|l|}{ Denmark } & \multirow{2}{*}{$\frac{1 \text { st factor }}{0.833}$} \\
\hline $\begin{array}{l}\text { Control/ } \\
\text { reciprocity }\end{array}$ & $\begin{array}{l}\text { Most unemployed people could find a job if they } \\
\text { really wanted to. }\end{array}$ & \\
\hline Attitude & $\begin{array}{l}\text { Unemployed people ought to be grateful to the } \\
\text { broader community because they receive benefits } \\
\text { and services. }\end{array}$ & 0.791 \\
\hline Identity & $\begin{array}{l}\text { I have little in common with typical unemployed } \\
\text { people. }\end{array}$ & 0.604 \\
\hline Need & $\begin{array}{l}\text { How do you estimate the living standard for } \\
\text { unemployed people in Denmark? }\end{array}$ & 0.508 \\
\hline Eigenvalue & & 1.943 \\
\hline & & 0.486 \\
\hline $\mathrm{N}$ & & 1405 \\
\hline \multicolumn{2}{|c|}{ The Netherlands } & 1st factor \\
\hline $\begin{array}{l}\text { Control/ } \\
\text { reciprocity }\end{array}$ & $\begin{array}{l}\text { How often do you think it is the case that } \\
\text { unemployed people make little or no effort to } \\
\text { find a job? }\end{array}$ & 0.729 \\
\hline Attitude & $\begin{array}{l}\text { Unemployed people ought to be grateful to the } \\
\text { broader community because they receive benefits } \\
\text { and services. }\end{array}$ & 0.787 \\
\hline Identity & $\begin{array}{l}\text { I have little in common with typical unemployed } \\
\text { people. }\end{array}$ & 0.678 \\
\hline Need & $\begin{array}{l}\text { To what extent do you think it is difficult to make } \\
\text { ends meet on unemployment benefits? }\end{array}$ & 0.378 \\
\hline Eigenvalue & & 1.753 \\
\hline $\mathrm{R}^{2}$ & & 0.438 \\
\hline $\mathrm{N}$ & & 1422 \\
\hline
\end{tabular}




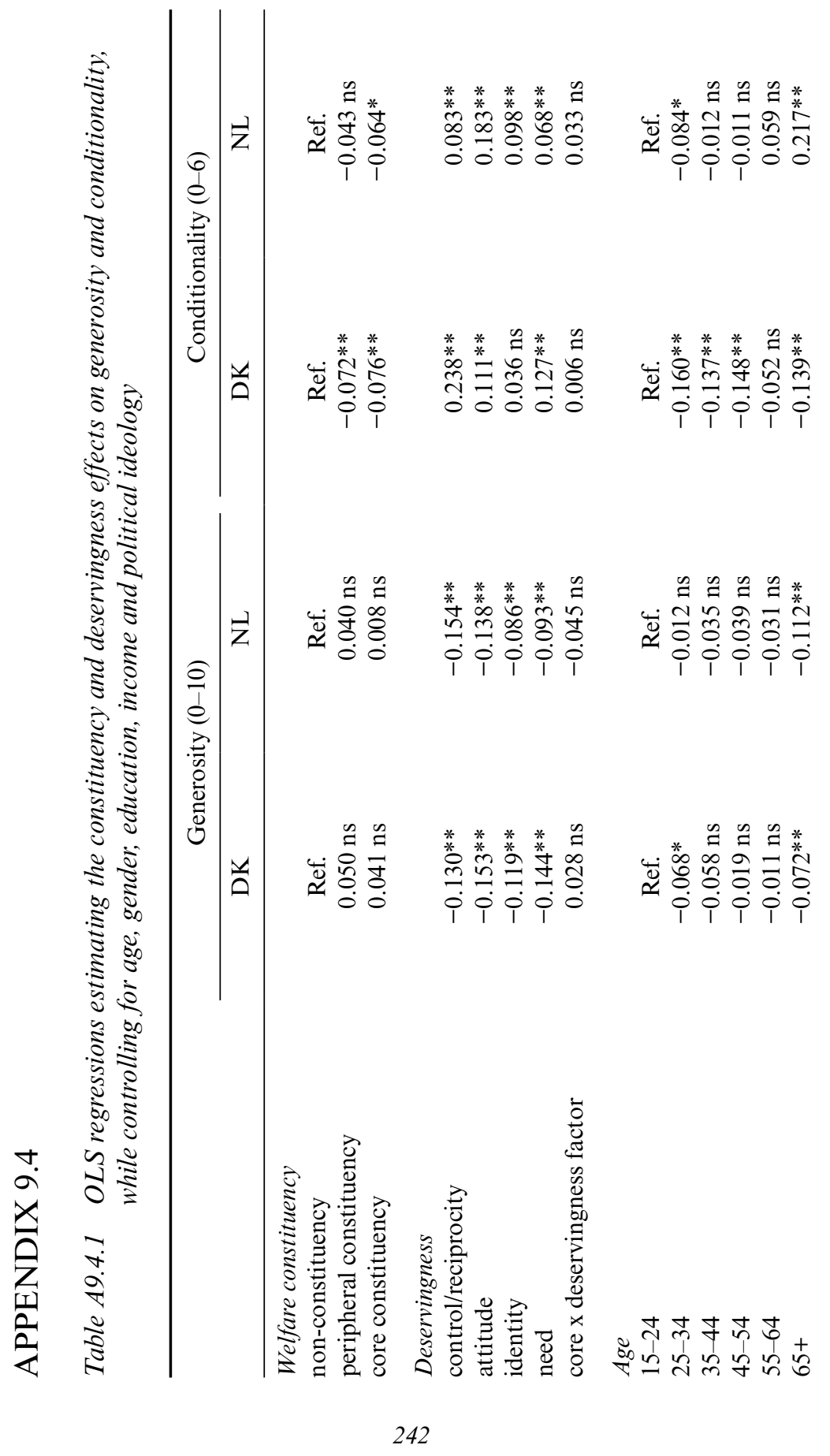




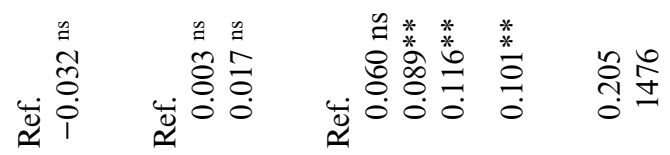

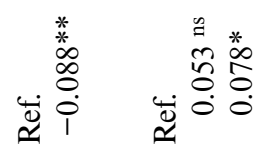

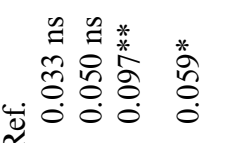

$$
\begin{aligned}
& \text { ปิ ڤ્ટ }
\end{aligned}
$$

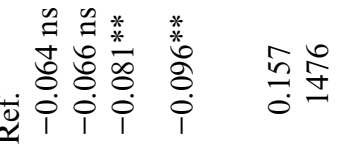

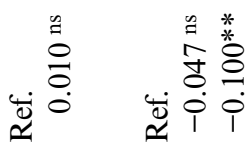

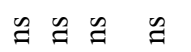

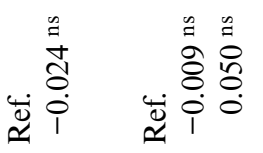

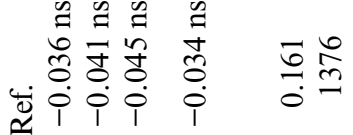

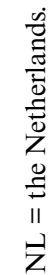

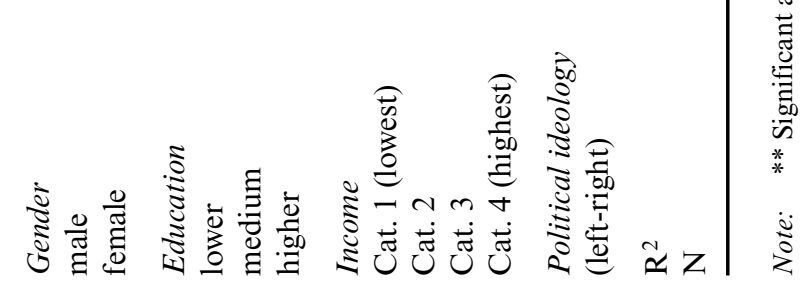




\section{APPENDIX 9.5}

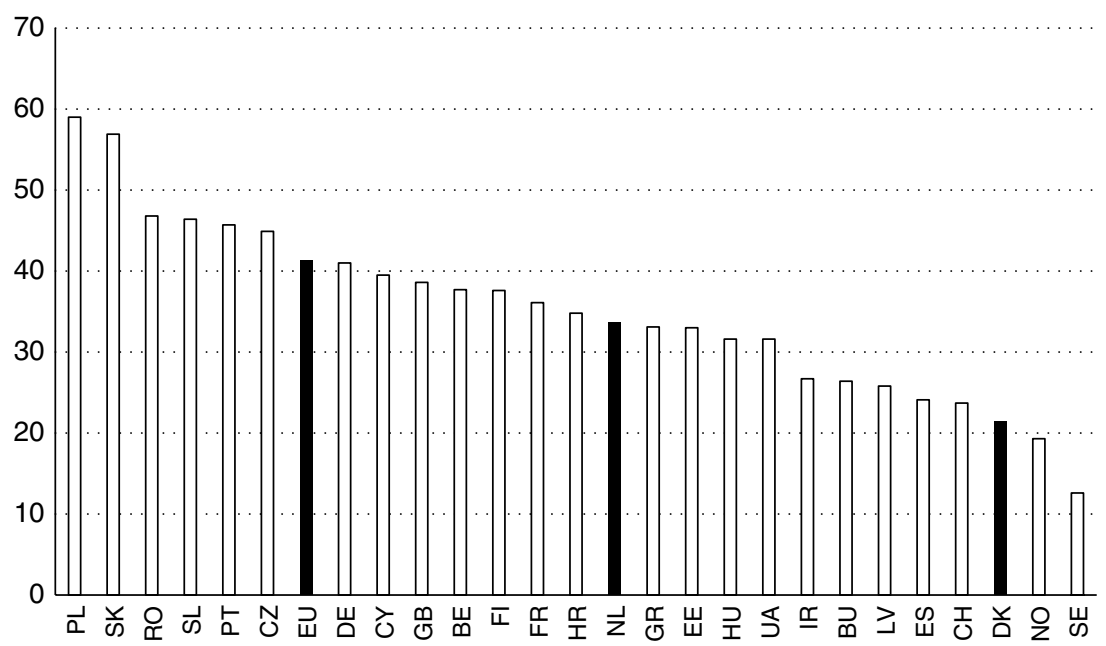

Note: The countries are: PL Poland; SK Slovakia; RO Romania; SL Slovenia; PT Portugal; CZ Czech Republic; DE Germany; CY Cyprus; GB Great Britain; BE Belgium; FI Finland; HR Croatia; NL the Netherlands; GR Greece; EE Estonia; HU Hungary; UA Ukraine; BU Bulgaria; LV Latvia; ES Spain; CH Switzerland; DK Denmark; NO Norway and SE Sweden. EU is the European average. Israel, the Russian Federation and Turkey were not included because they are generally not considered to be part of Europe. The question wording for experience with unemployment is: 'Have you ever been unemployed and seeking work for a period of more than three months in the past 5 years?' The question wording for the deservingness perception is: 'Please say how much you agree or disagree with the following statement about people in [country]. Most unemployed people do not really try to find a job'.

Figure A9.5.1 The proportion of ESS respondents (in \%) with experience of unemployment in the previous five years who agree (strongly) with the statement that 'most unemployed people do not really try to find a job' 